\title{
La Atención Primaria en Salud, Nuevos Enfoques y Perspectivas. Hallazgos desde un Programa de Formación y Capacitación
}

\author{
Alex Alarcón Hein ' \\ Adrián Torres Canales ${ }^{2}$ \\ Roberto Barna Yuri ${ }^{3}$ \\ Fecha Recepción: 30/07/2012 \\ Fecha aceptación: 20/08/2012
}

\section{Resumen}

El presente artículo presenta los principales hallazgos encontrados en la etapa de evaluación diagnóstica en el marco del programa de diplomado Enfoques y Nuevas Perspectivas para la Atención Primaria de Salud, realizado por la Escuela de Salud Pública de la Universidad de Chile en la Corporación Municipal de Valparaíso. Esta evaluación diagnóstica participativa, tuvo como objetivo el definir una línea base de trabajo y evaluación posterior. Asimismo, contribuyó a sentar las bases de lo que sería posteriormente el trabajo de tutoría para el desarrollo de un programa de intervención en salud en los Centros de Salud Familiar participantes de esta experiencia.

Palabras claves: Formación de Recursos Humanos en Salud, Atención Primaria de Salud, intervenciones.

\section{Abstract}

This article presents the main findings in the diagnostic evaluation stage under the diploma program New Approaches and Perspectives for Primary Health Care, held by the School of Public Health from the University of Chile in the Municipal Corporation of Valparaiso. This diagnostic and participatory evaluation aimed to define a baseline and subsequent evaluation work. It also helped to lay the groundwork for what would later be work tutoring in developing a health intervention program in Family Health Centers with those who participated in this experience.

Keywords: Human Resources Training in Health, Primary Health Care, interventions.

\footnotetext{
I.- Académico e Investigador Escuela de Salud Pública, Universidad de Chile. División de Políticas y Gestión en Salud. Coordinador de Extensión. Correo: aalarcon@med.uchile.cl

2.- Académico e Investigador Escuela de Salud Pública, Universidad de Chile. División de Políticas y Gestión en Salud.

3.- Investigador en Salud, experto en Atención Primaria de Salud.
} 


\section{Resumo}

O presente artigo apresenta as principais descobertas encontradas na etapa de evacuação diagnóstica no marco do programa do diplomado Enfoques e Novas Perspectivas para a Atenção Primaria de Saúde, realizado pela Escola de Saúde Pública da Universidade de Chile na Corporação Municipal de Valparaíso. Esta evacuação diagnóstica participativa teve como objetivo definir uma linha base de trabalho e avaliação posterior.Assim mesmo, contribuiu para sentar as bases do que seria posteriormente o trabalho de tutoria para o desenvolvimento de um programa de intervenção em saúde nos Centros de Saúde Familiar participantes desta experiência.

Palavras-Chave: Formação de Recursos Humanos em Saúde,Atenção Primaria de Saúde , Intervenções

Introducción

\section{Modelos de Atención de Salud}

Las políticas públicas deben lograr el bienestar de toda una sociedad. Su implementación involucra normas, provisión de bienes y servicios, fuentes de financiamiento, determinación de objetivos y formas de aplicación, evaluación y control. En el sector salud, estas acciones cada día se hacen más relevantes para el desarrollo del sector salud post reforma de 2004, lo que requiere situarse y prepararse para enfrentar fuertemente esta etapa.

En este escenario,se debe profundizar la relevancia queadquieren los cambios biopsicosociales a los que este proceso apunta. Es ahí donde la preparación de los profesionales de la salud pasa a constituir una pieza fundamental de todo el proceso de cambios.

A partir del proceso de Reforma de Salud vigente en Chile desde la promulgación de la Ley de Autoridad Sanitaria y Gestión en Red (Ley $N^{\circ}$ 19.937, 2004), se define que el modelo integral de salud con enfoque familiar constituye la base del sistema de salud del país.

Antes de entrar a analizar los aspectos específicos de este modelo y sus implicancias en el sistema de salud chileno, se debe hacer presente que los modelos, tal como los ideales, tienen un carácter coherente y perfecto que nunca se pueden cumplir (Capponi, 2004). Por eso, deben ser tomados como proposiciones a las que se aspira llegar, de carácter transitorio, que se modifican ante los nuevos aportes de las ciencias y del conocimiento humano. En este sentido, en este artículo se discutirán previamente algunos elementos que son comunes a la definición de modelos de salud, para después profundizar en las particularidades del modelo chileno actualmente vigente, $y$ que dan pie a la propuesta.

El término modelo de atención se refiere al modo cómo las instituciones formales de salud enfrentan y abordan el proceso salud-enfermedad y las distinciones que realizan del mismo (Hidalgo, 1999). Corresponde al elemento central de la estrategia sanitaria de un país y define la relación del sistema de salud con la población. Comprende, por tanto, la forma cómo se organizan los recursos para satisfacer la demanda de la población y el modo cómo se relacionan los distintos elementos del sistema para satisfacer las necesidades 
de cobertura en atención promocional, preventiva, curativa, de rehabilitación y cuidados paliativos.

El modelo de atención, al establecer principios ordenadores de la actividad asistencial, se materializa en una estructura organizacional que permite la interacción de la organización con los ciudadanos, prestadores y funcionarios de la salud, consigo misma y con otras instituciones u organizaciones. A su vez, la estructura organizacional define la forma de relación y coordinación del conjunto de establecimientos que constituyen la infraestructura sanitaria del país.

Un modelo de salud, en lo esencial, busca satisfacer las necesidades y expectativas del usuario respecto a mantenerse sano o saludable, mejorar su estado de salud actual en caso de enfermedad y garantizar su estado de salud futuro.

Se entiende por sistema de atención la organización del proceso, en el cual intervienen establecimientos de salud con diferentes niveles de complejidad (Martín-Zurro, 1993). El objetivo de producción del sistema es satisfacer las necesidades y expectativas de atención de las personas en salud, procurando optimizar las intervenciones y mejorar su eficacia y eficiencia.

El ordenamiento de los establecimientos de atención en salud, operando coordinadamente dentro de un territorio asignado, mediante vínculos institucionales o contractuales, es lo que se denomina trabajo en red asistencial. Estas redes incluirán, al menos, a los establecimientos que dependen del Servicio de Salud y los de atención primaria, sea ésta de administración municipal u otra.

Bajo el lema Salud para Todos, en la conferencia internacional de Alma Ata (OMS, 1978) se produjo un refuerzo del modelo de atención que privilegia la atención primaria, la participación comunitaria, el trabajo intersectorial y el uso de tecnologías apropiadas (ej.: solución ambulatoria de los problemas) en lo que se pasó a llamar la estrategia de atención primaria.

Esta recomendación, que surgió de los técnicos y del mundo político, se enfrentó con una tendencia mundial a la sobre especialización médica, al consumo excesivo de medicamentos y al abuso de tecnologías, muchas veces de eficacia dudosa. La crisis de la deuda externa en el inicio de los años ochenta y, posteriormente, la globalización de las economías, significó que los países reaccionaran a la explosión de costos que enfrentaban en el sector salud. Eso fue determinante en prefigurar la tendencia actual, en la que se han encontrado antiguos principios de la salud pública con el pragmatismo del pensamiento económico, dando como resultado un movimiento mundial que entrega cada vez más poder al usuario y a la atención primaria y que, por otro lado, incentiva el mínimo uso de los hospitales y de la complejidad tecnológica, exigiendo evidencias que respalden los cursos terapéuticos a seguir (Martínez O., 1999).

Lo señalado anteriormente a favor del usuario y de la atención primaria, se complementa con técnicas de gestión que separan la compra de atenciones de salud de la provisión de esas atenciones, velando los aseguradores por los derechos y satisfacción de los usuarios que se enrolan libremente con ellos. A la vez, los aseguradores se encargan de 
administrar sus cotizaciones o seguros de salud. Estas interrelaciones entregan también la capacidad de comprar prestaciones complejas (laboratorio, radiología). Para el control de los proveedores existe un ente regulador, normativo y fiscalizador, que cuida los intereses globales de los ciudadanos y de la sociedad. Esta función la cumple el Estado a través del Ministerio de Salud.

En Chile, un aspecto de la Reforma del sector salud se basó en separar las funciones descritas: Rol de Rectoría o Normativo (Ministerio de Salud); Rol de Fiscalización (Secretarias Regionales Ministeriales, Instituto de Salud Pública, Superintendencia de Salud); Rol de Prestador (Servicios de Salud; Establecimientos de Salud Municipalizados; Prestadores Privados).

En términos generales, los sistemas de salud que dan soporte al modelo de atención se estructuran básicamente de tres formas (A. Rico, 200I):

- Con criterios de mercado, en que la coordinación al interior del sistema está determinada por los precios, que se supone incorporan toda la información relevante para la toma de decisiones;

- Como estructura jerárquica, en que la coordinación se consigue recurriendo a planes y cursos de acción definidos por la autoridad que comanda el sistema; $y$

- Como redes asistenciales, en que la coordinación se logra a través de la negociación y acuerdos voluntarios entre actores interdependientes.

- Formas Mixtas, que combinan alguno(s) de los criterios anteriores.

En la actualidad, en Chile ha cobrado particular importancia la organización del modelo de atención sobre la base de Redes Asistenciales, al interior de las cuales pueden darse también modalidades jerárquicas de organización y mecanismos de mercado.

En relación a este tema se examinará la situación actual y sus principales insuficiencias, el Modelo de Atención deseable para la implementación de la Reforma de Salud y cuáles son las modificaciones necesarias de implementar para transitar desde la condición actual a la deseada.

Modelo de Atención Integral

El Ministerio de Salud, a partir de la Reforma de Salud, define el Modelo Integral de Salud (Ministerio de Salud, Depto.APS, 2002) como:

El conjunto de acciones que promueven y facilitan la atención eficiente, eficaz y oportuna que se dirige, más que al paciente o a la enfermedad como hechos aislados, a las personas, consideradas en su integralidad física y mental y como seres sociales pertenecientes a diversos tipos de familia, que están en un permanente proceso de integración y adaptación a su medio ambiente físico, social y cultural.

El Modelo de Atención Integral se dirige a la consecución de una mejor calidad de vida para las personas, las familias y las comunidades, con un énfasis en la anticipación de los problemas y el consiguiente acento en promoción de la salud y prevención de la enfermedad, es decir, anticipatorio al daño. 
Los aspectos de promoción de la salud y educación para lograr estilos de vida más saludables son competencias definidas para el equipo de salud en forma transversal, las que, progresivamente, deberán ser articuladas con el trabajo intersectorial impulsado por los gobiernos locales y regionales a través de Vida Chile y otras instancias intersectoriales. El desafío epidemiológico con que se inicia el segundo milenio obliga a devolver protagonismo a los ciudadanos y sus organizaciones en el cuidado de su salud, reorientando el rol del equipo de salud a uno asesor y cuidador, que acompaña el proceso salud - enfermedad (Ministerio de Salud, 2002).

El quehacer sanitario definido en este modelo de atención se basa en un equipo de salud multidisciplinario y de liderazgo horizontal, que se hace cargo de la población de un territorio acotado. Allí aplica el modelo de salud familiar, es decir, acompaña a los integrantes de la familia durante todo el ciclo vital, apoya sus momentos críticos con consejería, educación y estrategias de promoción de salud, detecta oportunamente el daño y proporciona asistencia clínica y rehabilitación oportuna, en caso de patología aguda o crónica.

Las instituciones de salud se organizan en la red asistencial con mecanismos establecidos de relación interinstitucional, reconociendo que el equipo de salud de cabecera es el encargado de brindar seguimiento a las personas y familias. La red asistencial se debe organizar de modo de poder dar respuesta oportuna e informar adecuadamente al equipo de salud de lo que sucede con una persona enferma, durante su derivación a otro de los establecimientos integrantes de ésta. Los niveles de mayor complejidad clínica deben, entonces, organizarse para responder a los requerimientos de la red asistencial en que están insertos.

En el modelo descrito, la comunidad y sus redes de autocuidado, y el nivel primario de atención, son el eje articulador del quehacer sanitario. Son, a su vez, el nivel que concentra la complejidad socio sanitaria.

\section{Modelo de Salud Familiar}

El modelo de Salud Familiar proporciona una nueva visión a la salud,ya que pone énfasis en la prevención y promoción de ésta desde un enfoque biopsicosocial, donde la responsabilidad del bienestar personal y social no recae solamente en los equipos de salud sino también en las propias comunidades.

Las características del modelo se encuentran claramente representadas en el documento Modelo de Atención Integral en Salud (Ministerio de Salud, op.cit.) y pueden resumirse como sigue:

I. Que esté centrado en el usuario, facilitando el ejercicio pleno de sus deberes y derechos en salud.

2. Que tenga un énfasis en lo promocional y preventivo, con enfoque familiar de los cuidados.

3. Que sea integral, tanto en la comprensión de los fenómenos como en las formas de afrontarlos.

4. Que garantice la continuidad de la atención, desde los cuidados primarios hasta las 
modalidades especializadas.

5. Que considere un trabajo en red, tanto sanitaria como social, que asegure la complementariedad que requieren las necesidades explícitas e implícitas en salud.

6. Que tienda al cuidado ambulatorio.

7. Que abra espacios para la participación en salud y la intersectorialidad.

8. Que promueva la mejora continua de la calidad de atención.

9. Que en todo momento considere una adecuada política de gestión de las personas que trabajan en el sector.

El enfoque,formulado desde un modelo biopsicosocial, es una forma de asumir la integralidad de las situaciones de salud/enfermedad de las personas y familias, incluyendo las distintas dimensiones del ser humano que permiten comprender la experiencia que se manifiesta en síntomas o signos.

La función, entonces, ya no es curar la enfermedad sino cuidar la salud. Se pasa de una medicina centrada en la patología a una atención focalizada en la persona y su particular forma de vida, su contexto familiar y social. Así, el rol de los profesionales se amplía a la promoción activa de la salud a lo largo de la vida del grupo familiar, asumiendo una función de facilitador y reconociendo a este como responsable y a cargo de su propia salud.

Finalmente, cabe señalar que el cambio del paradigma del modelo de atención de un enfoque biomédico, que prevaleció por largos años en nuestro país, a un modelo de atención integral de salud familiar, ha implicado grandes cambios en la estructura organizacional de cada establecimiento de Atención Primaria de Salud (APS). Para implementar esta reforma se ha hecho necesario introducir modificaciones en el funcionamiento de los establecimientos APS en diversos aspectos, tales como: financiamiento, capacitación, recurso físico, humano, de infraestructura, administrativo y en general en la forma de trabajo que han desarrollado los funcionarios de la salud por largo tiempo y que ya no da respuesta adecuada a los actuales desafíos; en esta línea, se han formado equipos interdisciplinarios a cargo de una población asignada.

Todos estos cambios y exigencias han tenido gran impacto en los trabajadores de la salud y en los ambientes laborales, lo que se manifiesta a través de una sensación de insatisfacción, por la falta de políticas que promuevan en forma clara y explícita el cuidado de quienes entregan la atención en salud.

\section{Características del modelo}

El modelo de atención se caracteriza por los siguientes elementos:

a) Enfoque biopsicosocial, con énfasis en la familia y comunidad. El mayor desafío del sistema se encuentra en el desarrollo de habilidades y destrezas que permitan a los equipos de salud analizar el proceso salud-enfermedad centrándose en las personas y desde una perspectiva sistémica. En este enfoque, se entiende a la familia como el sistema social primario de las personas, lo que implica introducirla como unidad de atención, con una oferta específica de prestaciones cuando así lo necesiten o como factor protector y terapéutico para los individuos. 
Las enfermedades crónicas, con compromiso multiorgánico, se constituyen en la actualidad como el grupo de patologías más relevante, tendencia que se consolidará en el futuro. Frente a esta situación, la organización de la atención por especialidades médicas redunda en que las personas acudan a tantos especialistas como distintos problemas de salud presenten. Lo anterior se traduce en la reproducción del paciente en el sistema especializado y en el incremento del tiempo destinado por las personas a atender sus problemas de salud, lo que puede ser causa de sufrimiento, especialmente en los casos más severos. Cabe destacar que esta forma de atención incide en la alta correlación que existe entre comorbilidad y costo.

Estudios realizados señalan que la distribución del costo de atención de salud es la siguiente (Hopkins, 1998):

\begin{tabular}{|ll|}
\hline$\%$ de la Población & $\%$ del Costo \\
\hline $1 \%$ & $30 \%$ \\
\hline $10 \%$ & $72 \%$ \\
\hline $50 \%$ & $97 \%$ \\
\hline
\end{tabular}

Cabe destacar que de acuerdo a investigaciones de modelos de ajuste de riesgo la mayoría de casos de alto costo son producto de comorbilidad (Ellis, 2004). De lo anterior se desprende la necesidad de modificar la estrategia de atención a las personas con múltiples problemas de salud.

b) Continuidad de la atención y de los cuidados de salud. La continuidad de cuidados de salud se extiende a toda la red a través del trabajo en red (asistencial, comunitaria, social). En el nivel primario, la red estará dada por una forma organizacional que permita una relación cercana con una población a cargo (sectorización). Este equipo conoce a su comunidad de usuarios en los aspectos de calidad de vida, factores de riesgo y factores protectores; dispone, por lo tanto, de elementos para actuar con estrategias preventivas y promocionales adaptadas a esa realidad.

c) Prestación de servicios integrados. A pesar de que tradicionalmente se habla de consultas y controles, se espera que en cada contacto con individuos y familias se visualice la oportunidad para incorporar acciones de promoción y prevención, fomentando el autocuidado individual y grupal. Este equipo de salud debe ser capaz de actuar con servicios integrados y acordes a las particularidades socioculturales de la población usuaria, especialmente en torno de los problemas psicosociales prevalentes y emergentes en la población.

d) Resolutividad. Se traduce en la capacidad del equipo de salud para contribuir al cuidado de la salud de las personas de su sector, sumada a la capacidad diagnóstica y excelencia clínica. Cada establecimiento debe disponer del equipamiento y de los recursos humanos calificados para abordar los desafíos referidos a evitar el daño en salud, pesquisarlo precozmente, confirmar diagnósticos, tratar enfermedades de acuerdo a los protocolos y cumplir su rol en la red asistencial. Particularmente, en el ámbito psicosocial, el equipo de salud de atención primaria ha debido ampliar sus competencias para abordar la problemática 
emergente, compleja y diversa que enfrentan las familias y comunidades.

e) Responsabilidad compartida entre todos los miembros de la familia y la comunidad por la salud familiar y de cada uno de sus integrantes. Se promueve la participación de la familia, tanto en las actividades preventivo-promocionales, como en las recuperativas. En especial, se favorece la participación de la pareja en el proceso reproductivo; del padre y la madre, en el cuidado de los hijos; de algún integrante de la familia y comunidad en el cuidado de las personas con enfermedades crónicas, adultos mayores, discapacitados, personas postradas o en fase terminal de alguna enfermedad.

\section{El Modelo de Gestión en Red}

La organización de la atención de salud en Chile, desde los orígenes del Servicio Nacional de Salud (Ministerio de Salud, I98I), se caracterizó por su estructura planetaria, donde para un territorio determinado existía un establecimiento base con el cual se relacionaban los establecimientos del territorio. En este diseño, los márgenes del sistema estaban constituidos por Consultorios Urbanos y Rurales y Postas Rurales, establecimientos con una estructura organizacional simple, lo que los hacía fácilmente accesibles a la población; estos debían resolver problemas médicos de baja complejidad y con una alto porcentaje de cobertura.Ante problemas de salud que sus operadores estimaran que no podían resolver, estos establecimientos derivaban a los pacientes a establecimientos de mayor complejidad organizacional. La mayor complejidad organizacional correspondía a hospitales con muchas especialidades y a los centros de referencia nacional para temas específicos como, por ejemplo, el Instituto de Neurocirugía o el Instituto del Tórax. Estos establecimientos se caracterizaban, además, por una alta complejidad bio-médica y tecnológica, y una baja cobertura poblacional.

Un concepto complementario al anterior, que jerarquizaba organizacionalmente a los establecimientos, tenía que ver con los niveles de complejidad médica de los problemas de salud que debía resolver cada establecimiento, lo que determinaba sus funciones específicas. Así, se distinguieron:

- El nivel primario, lo más cercano al paciente, su puerta de entrada al sistema de atención, localizándose allí todo el esfuerzo preventivo y de fomento de la salud, con un fuerte componente intersectorial y curativo, basado en la atención brindada por un equipo multiprofesional.

- El nivel secundario o de especialidades, constituido por la atención de especialistas, usualmente en el Consultorio Adosado de Especialidades (CAE) ○ los Centros de Referencia en Salud (CRS), asociados a hospitales complejos, esencialmente centros de derivación donde se concentran recursos escasos del sistema; $y$

- El nivel terciario u hospitalario, al que se recurre sólo cuando la gravedad del cuadro o su complejidad requieren que el paciente esté en observación permanente o requiera una intervención mayor.

La revolución tecnológica y de las comunicaciones ha introducido nuevos cambios en el sistema y en la actitud de los usuarios. Por ello, la tendencia actual es hablar de atención ambulatoria y atención hospitalaria, conjugando de mejor forma los principios de la salud 
pública con los de la administración eficiente. Las metas sanitarias para la década, sumadas a los cambios descritos, permiten pensar en complejizar y mejorar la integración en red al nivel primario, además de promover la entrega creciente de poder al usuario en toda la red asistencial. La tendencia derivada de este cambio de ejes debiera traducirse en un uso de los hospitales decreciente en cantidad, pero creciente en complejidad.

Se entiende por sistema de atención la organización del proceso, en el cual intervienen establecimientos de salud con diferentes niveles de complejidad (Martín-Zurro, 1993). El objetivo de producción del sistema es satisfacer las necesidades y expectativas de atención de las personas en salud, procurando optimizar las intervenciones y mejorar su eficacia y eficiencia. La constatación de las características anteriores y la búsqueda de estrategias para intervenir sobre ellas, son un desafío cuando lo que se propone en el Modelo de Gestión en Red es dar paso a la conformación de la red como la unidad básica de coordinación entre los distintos niveles de atención, que le permitan al usuario transitar por ellas con el fin de obtener la oportuna detección y resolución de sus problemas de salud.

Frente a la enfermedad o a la sospecha de ésta y superada la capacidad resolutiva del equipo de salud de atención primaria, el usuario es derivado a la atención del nivel secundario de atención, que se ejerce en los Centros de Referencia de Salud (CRS), Centros de Diagnóstico y Tratamiento (CDT) u otras formas de organización ambulatoria. Este nivel de atención se organiza para resolver en forma ambulatoria todo aquello que sea posible en el contexto de la realidad de su red asistencial y su misión está centrada en establecer el diagnóstico y resolver la patología de acuerdo a la realidad de cada especialidad. Se pone énfasis en la resolución quirúrgica oportuna, la compensación precoz de cuadros crónicos (seguida de la devolución asesorada para manejo por parte del equipo de salud familiar si procede) y el acompañamiento de la rehabilitación y reinserción de personas con secuelas.

Este nivel primario de atención adopta estrategias destinadas a mantener a los equipos de salud capacitado para que las personas enfermas reciban sus tratamientos en forma continua y oportuna, sean controladas oportunamente y retomen el contacto con su equipo de salud de atención primaria frente a cualquier nueva problemática de salud.

Por su parte, el nivel secundario programa su oferta e identifica las brechas existentes, define estrategias para resolverlas y en aquellos casos que requiere apoyo para su resolución, las presenta al Consejo Integrador de Red Asistencial (CIRA), el cual analiza las necesidades y propone al Gestor de la Red Asistencial alternativas para demandar los recursos necesarios y equilibrar la oferta.

Finalmente, la atención hospitalaria se organiza para resolver la patología de mayor complejidad biomédica, electiva o de urgencia, busca la resolución ambulatoria de todo aquello posible en el contexto de la red asistencial, se responsabiliza por los resultados de los tratamientos efectuados y por la devolución oportuna del paciente al nivel apropiado de la red asistencial, manteniendo informado al equipo de salud de cabecera. Debe poner todos los recursos posibles al servicio de la actividad ambulatoria del nivel secundario, apoyado en una programación racional del uso de sus recursos.

Se espera que los equipos de salud que interactúan en el continuo de la red asistencial 
tengan como centro de su quehacer al usuario, debiendo poner el énfasis en la calidad de la atención y en la relación y trato con las personas. Su orientación debiera ser a la resolución oportuna de los problemas de salud tanto como a la prevención de la enfermedad.

La programación basada en el diagnóstico local representa uno de los procesos de mayor relevancia dentro de la gestión del sector salud. Ella permite orientar la utilización de los recursos disponibles hacia metas y resultados explícitos, buscando las mejores alternativas de acción frente a los desafíos que significa mejorar la situación de salud de las personas y contribuir a mejorar las condiciones de vida de la población. Para que la programación alcance su máxima efectividad como herramienta de apoyo a la gestión, es necesario tener en cuenta los principales elementos de contexto que inciden de manera más fuerte en ella.

El ordenamiento de los establecimientos de atención en salud, operando coordinadamente dentro de un territorio asignado, mediante vínculos institucionales o contractuales, es lo que se denomina trabajo en red asistencial. Las redes incluirán, al menos, a los establecimientos que dependen del Servicio de Salud y los de la atención primaria, sea ésta de administración municipal u otra.

\section{Material y Método}

El material utilizado para este trabajo ha sido recopilado durante el desarrollo del diplomado Enfoques y Nuevas Perspectivas para la Atención Primaria de Salud, realizado por la Escuela de Salud Pública de la Universidad de Chile en la Corporación Municipal de Valparaíso, entre el 18 de Junio de 2010 y el 22 de Enero de 201 I en el que participaron 27 profesionales de la Corporación Municipal de Valparaíso (Cormuval) y profesionales del departamento de Salud de la Municipalidad de San Antonio. Dichos profesionales cumplen labores profesionales en los Centros de Salud Familiar Las Cañas, Cordillera, Reina Isabel II y 30 de Marzo, además de establecimientos APS de la provincia de San Antonio.

El material fue parte de una evaluación diagnóstica realizada al comienzo del Programa, con el objetivo de establecer una línea base de trabajo y evaluación posterior. Asimismo, que permitiera sentar las bases de lo que sería posteriormente el trabajo de tutoría para el desarrollo de un programa de intervención.

Para realizar la evaluación diagnóstica se dividió el curso en cinco grupos de profesionales, que tuvieran similares conocimientos y aproximación a alguno de los centros elegidos para el trabajo de intervención y se les consideró un panel de expertos (Hernández S., Fernández-Collado, \& Baptista L., 2008) Los instrumentos elegidos para la evaluación diagnóstica, tanto por la accesibilidad y sencillez, como por la confiabilidad de los mismos, fueron en primer lugar la Matriz de riesgo ${ }^{16}$, para identificar las principales áreas críticas, $y$ posteriormente el Diagrama de Causa y Efecto o de Ishikawa ${ }^{17}$, para determinar las causas de los efectos-problema identificados en la Matriz.

16.- La Matriz de Riesgo utilizada es la de común uso en los Servicios de Salud chilenos y también usado por el Consejo General de Auditoría Interna Gubernamental.

17.- Para estos efectos se usó la cartilla del Diagrama de Causa y Efecto (Cause \& Efect Diagram), material de la Sociedad Latinoamericana para la Calidad. 
Cada grupo estuvo compuesto por alrededor de cinco profesionales de la Salud quienes, en conjunto con sus tutores, elaboraron un plan de trabajo. El método utilizado fue de carácter cuali-cuantitativo y comenzó con una pregunta abierta ${ }^{18}$ (Armas M.,y otros, 20I0) que se hizo para la elaboración de la Matriz de riesgo. Dicha pregunta fue la siguiente:

Describa, brevemente: ¿Cuáles son, a su juicio, los cuatro principales elementos de riesgo, que tiene el lugar en donde usted trabaja, actualmente, desde el punto de vista de la gestión?". Priorice de uno (I) a cuatro (4).

Posteriormente, guiados por un profesor, los participantes respondieron individualmente, primero, y consensuaron después, un listado de áreas críticas; realizaron una conciliación semántica de los contenidos y votaron por las distintas alternativas, utilizando un cuestionario de escala Likert (Hernández S., Fernández-Collado, \& Baptista L., 2008) hasta llegar a elaborar dos tablas donde evaluaron tanto la probabilidad de riesgo de ocurrencia como la importancia de los elementos descritos. Con esa información de insumo se llegó a elaborar la Matriz que contiene los principales elementos de riesgo, de acuerdo a la percepción de los profesionales consultados que para los efectos de este trabajo se denomina Panel de Expertos.

La matriz con los resultados, que se muestra más adelante en la Tabla $\mathrm{N}^{\circ} \mathrm{I}$, se elaboró de la siguiente manera:

Se volcaron los resultados de las tablas parciales, tanto de probabilidad de ocurrencia, medida en el cuestionario de escala Likert con una evaluación de uno (I) a cinco (5), como la de importancia medida en dicho cuestionario con una evaluación de uno (I) a diez (10). A cada tabla se le asignó un peso específico conforme a la experiencia de los tutores, asignándole a la primera tabla un peso de 0,4 y a la segunda un peso de 0,6. Al aplicar este factor de ponderación a las evaluaciones producto del trabajo consensuado del panel, arrojó como resultado un determinado Promedio Ponderado, cuya fórmula es la siguiente: $(\operatorname{Pr~Oc} * 0,4+\operatorname{Imp} * 0,6)=$ Prom Pond. ${ }^{19}$ Una vez realizado este ejercicio se listó de mayor a menor los distintos elementos surgidos del análisis de los grupos.

Adicionalmente, se agregó una columna para asignarle un determinado porcentaje dentro del contexto de cada grupo, cuya fórmula es: \% Si $(\mathrm{PPI}=100)$ entonces $(\mathrm{PPR}=\mathrm{X})$ Así $\%=(\mathrm{PPR} * 100 / \mathrm{PPI}) .{ }^{20}$ Finalmente, la última columna refleja el ámbito al que se vincula el elemento de análisis descrito en Breve descripción del riesgo.

\section{Tabla $\mathrm{N}^{\circ} \mathrm{I}$}

Descripción de Riesgos

SOME = Servicio de Orientación Médica y Estadística; CES= Centro de Salud; RRHH = Recursos Humanos. Fuente: Elaboración propia. Diplomado Enfoques y Nuevas Perspectivas para la Atención Primaria de Salud, (20I0)

18.- Este método ya fue usado antes; ver Armas M., y otros (2010).

19.- Donde $\operatorname{Pr}$ Oc = Probabilidad de Ocurrencia del elemento de análisis; Imp = Importancia del elemento de análisis; Prom Pon = Promedio Ponderado de los factores multiplicados por sus respectivos pesos específicos.

20.- Donde PPI = Promedio Ponderado Ideal y PPR = Promedio Ponderado Real . 


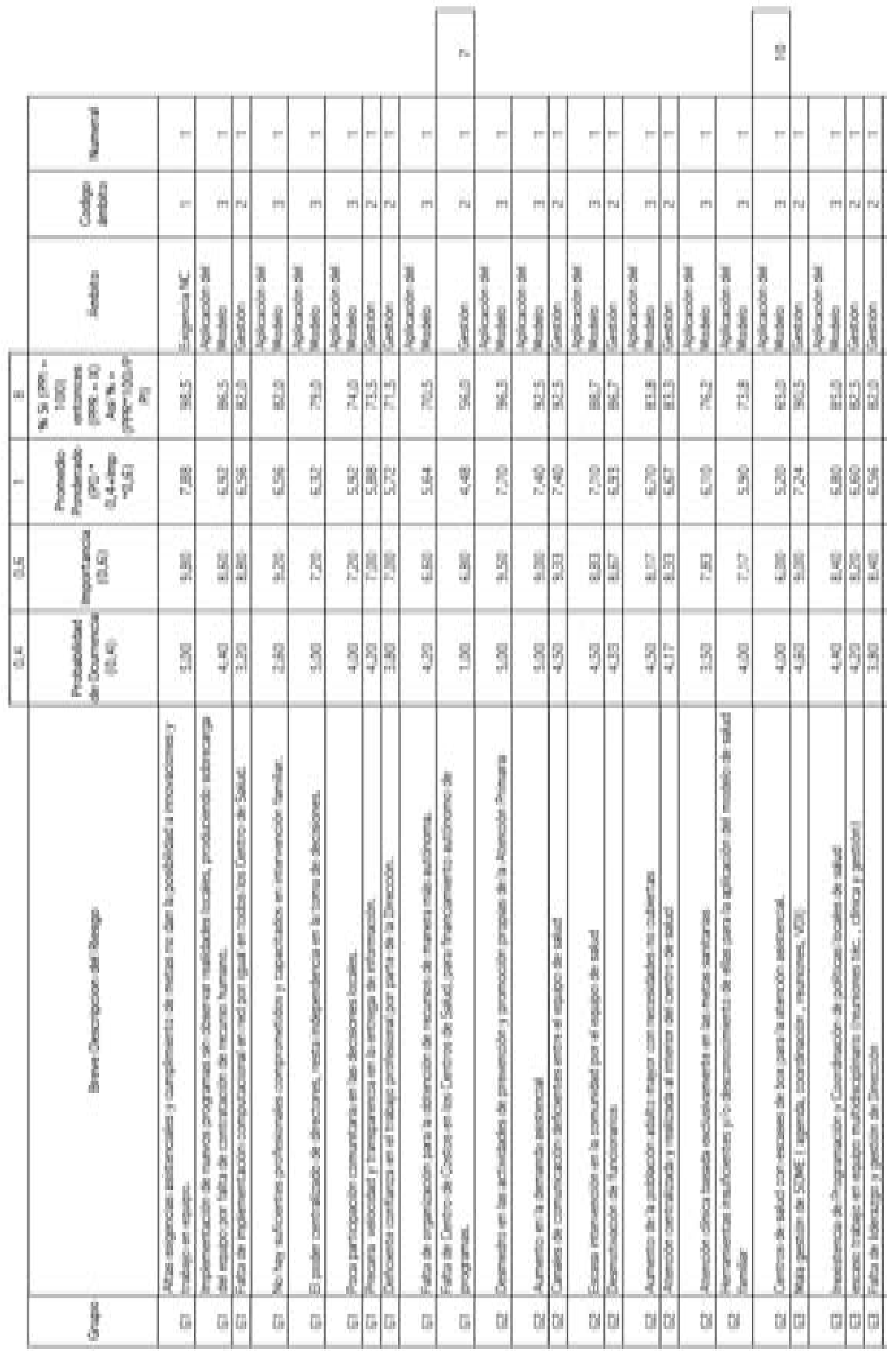




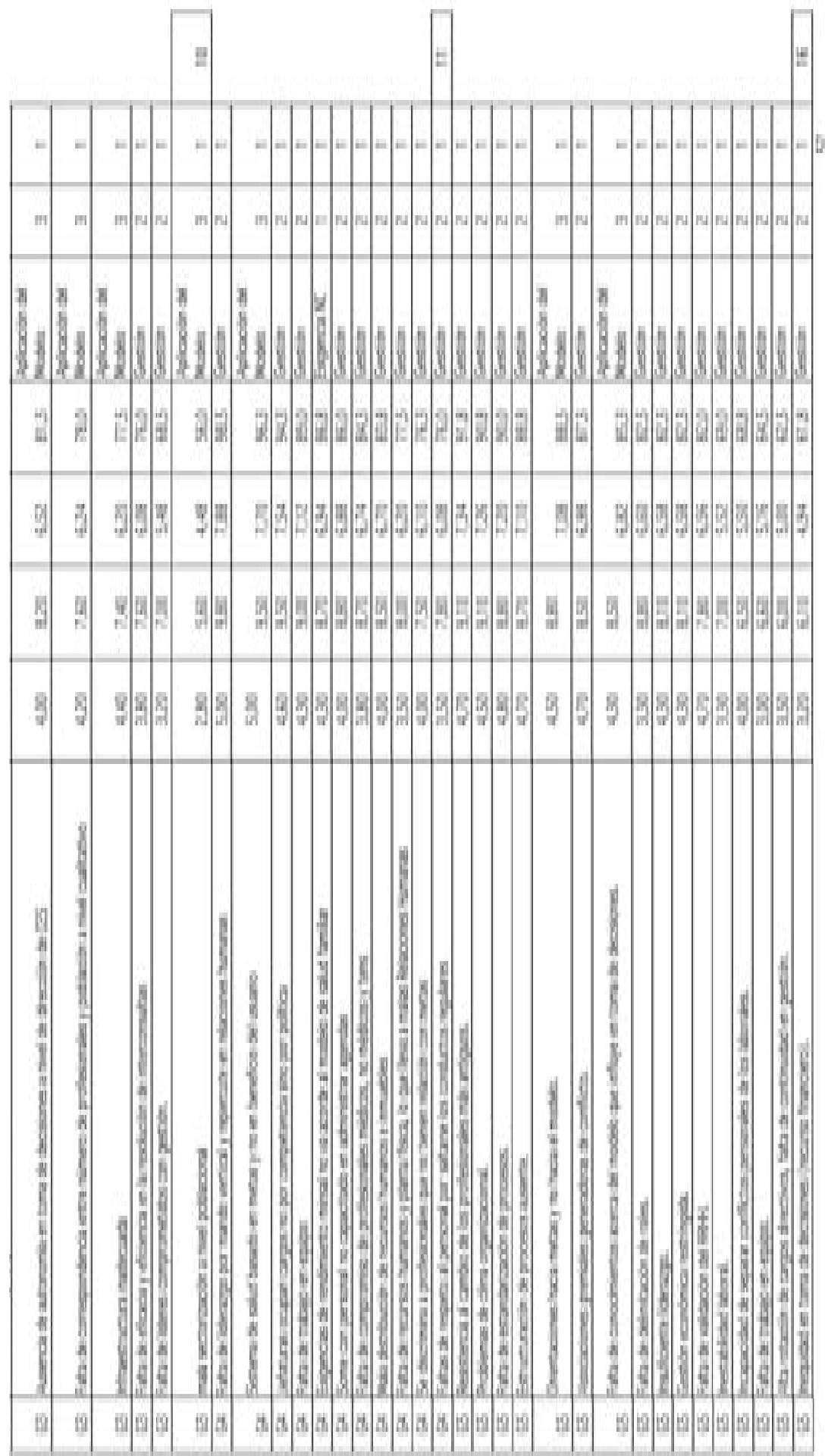


Posteriormente, los distintos grupos de participantes volvieron a analizar los resultados de la Matriz de Riesgo y eligieron de entre los problemas detectados tres que a juicio de ellos eran los más críticos y, por lo tanto, aptos para ser abordados y llevarlos al Diagrama de Causa y Efecto, con el objetivo de identificar lo que a su juicio eran las principales causas del problema detectado.

Los problemas llevados al Diagrama de Causa y Efecto o de Ishikawa fueron debidamente priorizados y a cada uno se le asignó un determinado peso específico ${ }^{21}$ dentro del conjunto con el propósito de identificar los puntos débiles para futuras planificaciones de intervención. Asimismo, cuando se trabajó en torno a la identificación de las causas de dichos problemas, a cada una de las causas y sub causas, también se les asignó un determinado peso, también con el propósito de afinar el diagnóstico,y plantear los futuros trabajos de intervención lo más afinadamente posible. Cabe destacar que el resultado de las distintas fases del proceso se obtuvo por consenso del Panel de Expertos, en aproximaciones sucesivas y utilizando una modalidad cuali-cuantitativa. (Konow \& Pérez, 1990)

\section{Resultados}

A continuación se presentan los resultados del trabajo realizado a partir de los diagramas de Ishikawa realizado por cada uno de los cinco grupos de participantes que trabajaron en el contexto del Diploma Enfoques y Nuevas Perspectivas para la Atención Primaria de Salud ya presentado. Cabe recordar que esta evaluación diagnóstica tuvo como objetivo definir una línea base de trabajo y evaluación posterior. Asimismo, contribuyó a sentar las bases de lo que sería posteriormente el trabajo de tutoría para el desarrollo de un programa de intervención en salud en los Centros de Salud Familiar, nivel Atención Primaria en Salud (APS), participantes de esta experiencia.

Cada grupo eligió las tres áreas más críticas detectadas a partir de la Matriz de Riesgo y las trabajó en un diagrama de Causa y Efecto, de manera de analizar cuáles eran las principales causas de cada área problema. Los resultados más relevantes por grupo fueron los siguientes:

\section{I Áreas Críticas de APS por Grupo}

GRUPO A. Las áreas más críticas de la APS, vistas desde su lugar de trabajo, fueron: $1^{\circ}$ El Modelo de Salud en la APS, $2^{\circ}$ Compromiso con el Modelo de Salud Familiar, y $3^{\circ} \mathrm{La}$ participación Comunitaria. Dentro de cada una de estas áreas - problema, las causas y respectivos pesos relativos dentro del diagrama se dio de la siguiente manera:

$I^{\circ}$ El Modelo de Salud en APS (Gráfico NI), se explica por las siguientes causas: Gestión y Políticas Públicas (40\%), Financiamiento (25\%), RRHH (I5\%), Instrumentos (5\%), Métodos de trabajo (5\%), Infraestructura (5\%), Comunicaciones (5\%). 
Gráfico $\mathrm{N}^{\circ} \mathrm{I}$ :

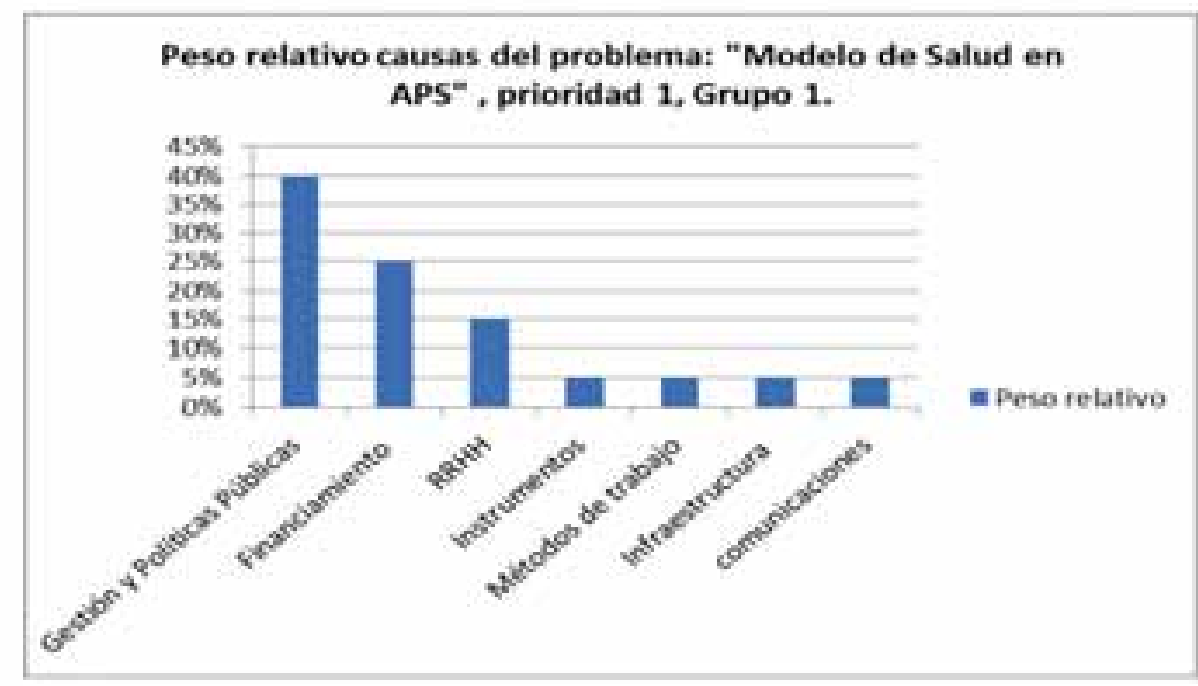

Fuente: Elaboración propia. Diplomado Enfoques y Nuevas Perspectivas para la Atención Primaria de Salud, (2010).

$2^{\circ}$ Compromiso con el Modelo de Salud Familiar (Gráfico №2), se explica por las siguientes causas: Financiamiento (30\%), RRHH (25\%), Métodos de trabajo (20\%), Clima Organizacional (15\%), Infraestructura (10\%).

\section{Gráfico $\mathrm{N}^{\circ} 2$ :}

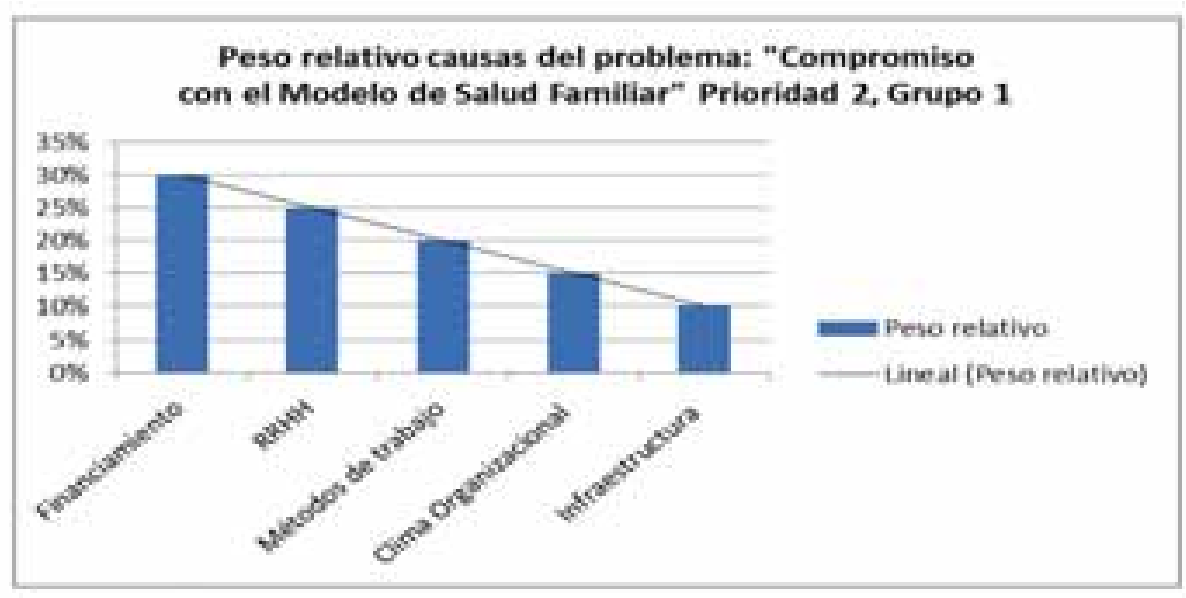

$3^{\circ}$ Participación Comunitaria (Gráfico N³), se explica por Políticas Públicas (25\%), Procesos (20\%), RRHH (I5\%), Financiamiento (I5\%), Comunidad (I5\%), Instrumentos (5\%), Infraestructura (5\%). 


\section{Gráfico $\mathrm{N}^{\circ} 3$ :}

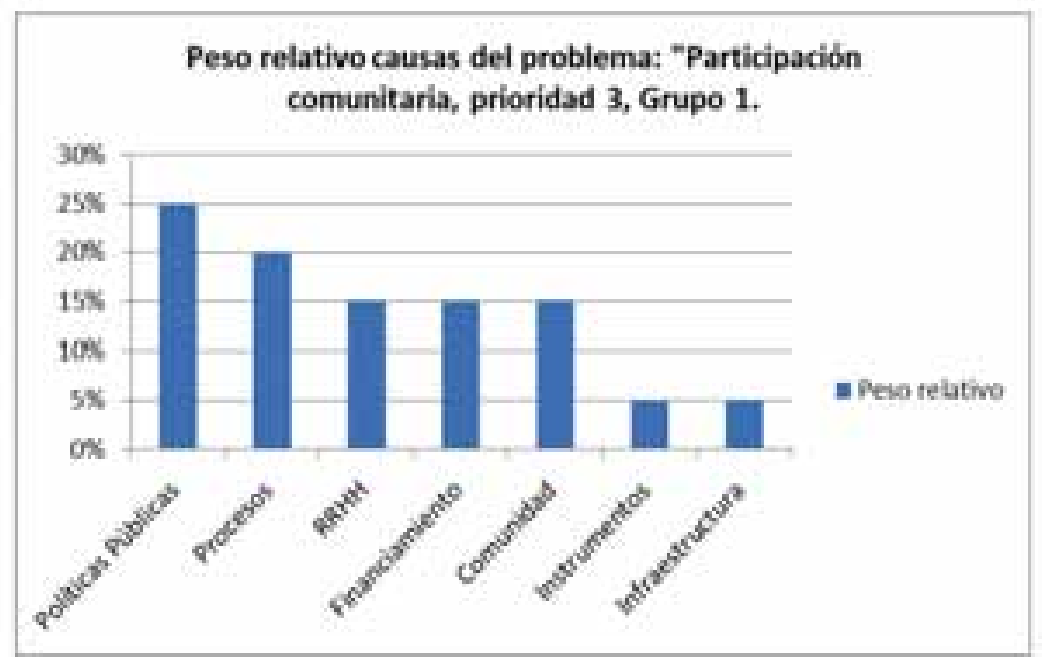

Fuente: Elaboración propia. Diplomado Enfoques y Nuevas Perspectivas para la Atención Primaria de Salud, (20I0).

GRUPO B. Las áreas más críticas de la APS priorizadas fueron: $1^{\circ}$ Incompatibilidad del Modelo de Salud Familiar con la realidad de la APS, $2^{\circ}$ Escasa participación e intervención comunitaria y $3^{\circ}$ Clima organizacional en APS.

Dentro de cada una, el análisis de las causas y respectivos pesos relativos dentro del diagrama se dio de la siguiente manera:

$I^{\circ}$ Incompatibilidad del Modelo de Salud Familiar con la realidad de la APS (Gráfico N4), se explica por las siguientes causas: Modelo (23\%), Desarrollo Organizacional (23\%), RRHH (17\%), Comunidad (13\%), Gestión Administrativa (13\%), Infraestructura (I I\%).

\section{Gráfico $N^{\circ} 4$ :}

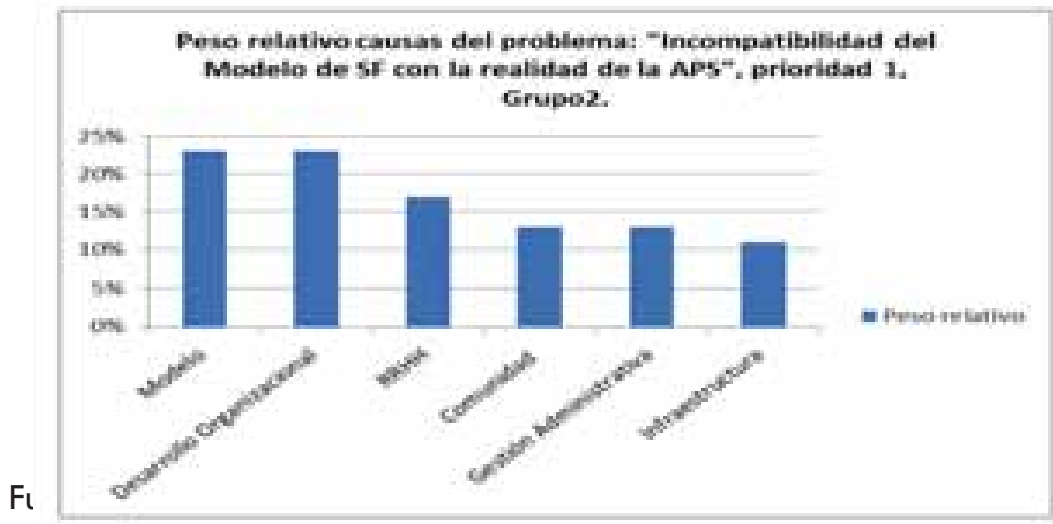

la Atención

Primaria de Salud, (20I0). 
$2^{\circ}$ Escasa participación e intervención comunitaria (Gráfico $\mathrm{N}^{\circ} 5$ ), se explica por las siguientes causas: Políticas de Salud (20\%), Gestión Administrativa (20\%), RRHH (19\%), Comunidad (I5\%), Infraestructura (14\%), Recursos Físicos y Materiales (12\%).

\section{Gráfico $N^{\circ} 5$ :}

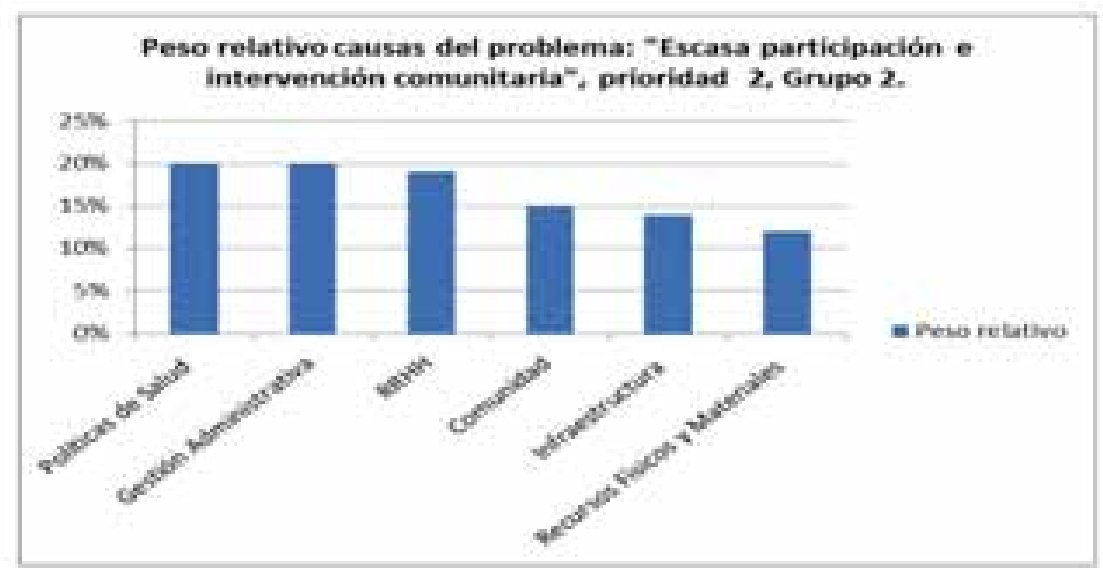

Fuente: Elaboración propia. Diplomado Enfoques y Nuevas Perspectivas para la Atención Primaria de Salud, (2010).

$3^{\circ}$ Clima organizacional en APS (Gráfico $N^{\circ} 6$ ), se explica por las siguientes causas: $\mathrm{RRHH}$ (28\%), Gestión Administrativa (20\%), Políticas de Salud (19\%), Gremios (I2\%), Comunidad (I I\%), Infraestructura (10\%).

\section{Gráfico $\mathrm{N}^{\circ}$ 6:}

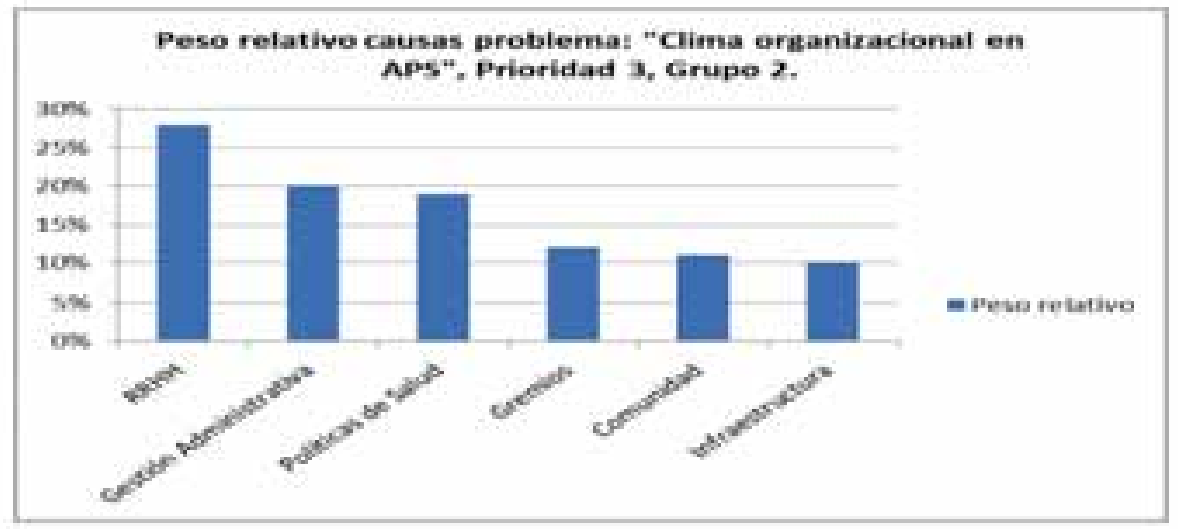

Fuente: Elaboración propia. Diplomado Enfoques y Nuevas Perspectivas para la Atención Primaria de Salud, (2010).

GRUPO C. Las áreas más críticas de la APS para este grupo fueron: $1^{\circ}$ Escasa Gestión de SOME, $2^{\circ}$ Políticas locales de Salud, y $3^{\circ}$ Enfoque de equipo multidisciplinario. 
$1^{\circ}$ Escasa Gestión de SOME (Gráfico $N^{\circ} 7$ ), la cual se explica por las siguientes causas: Gestión Administrativa (29\%), RRHH (27\%), Recursos Materiales (24\%), Planta Física (20\%).

\section{Gráfico N7:}

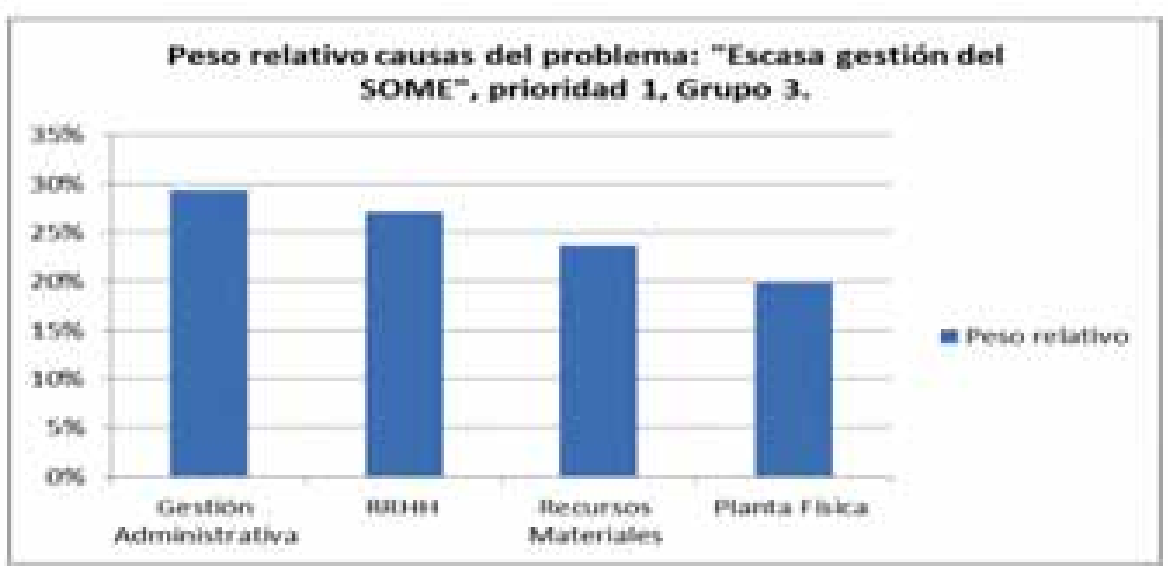

Fuente: Elaboración propia. Diplomado Enfoques y Nuevas Perspectivas para la Atención Primaria de Salud, (20I0).

$2^{\circ}$ Políticas locales de Salud (Gráfico $N^{\circ} 8$ ), se explica por las siguientes causas: Gestión Administrativa (22\%), RRHH (21\%), Comunidad (21\%), Recursos Materiales (18\%), Institucionalidad Local (18\%).

\section{Gráfico $\mathrm{N}^{\circ} 8$ :}

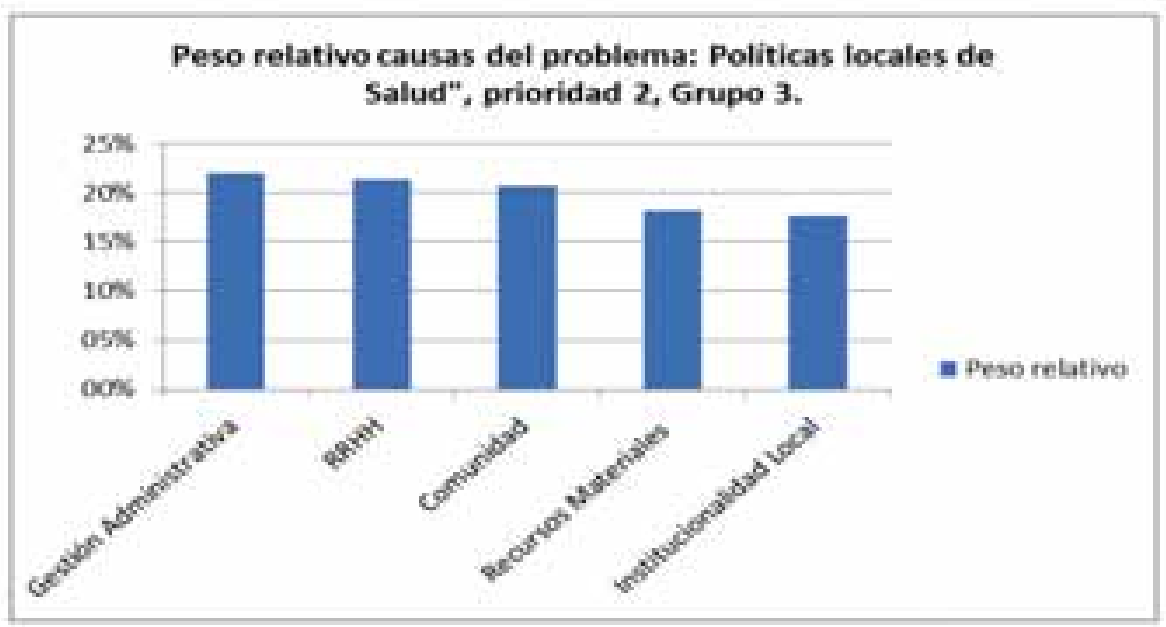

Fuente: Elaboración propia. Diplomado Enfoques y Nuevas Perspectivas para la Atención Primaria de Salud, (20I0). 
$3^{\circ}$ Enfoque de equipo multidisciplinario (Gráfico $\mathrm{N}^{\circ} 9$ ), se explica por las siguientes causas: RRHH (28\%), Gestión Administrativa (27\%), Planta Física (23\%), Recursos Materiales $(22 \%)$.

Gráfico $N^{\circ} 9$ :

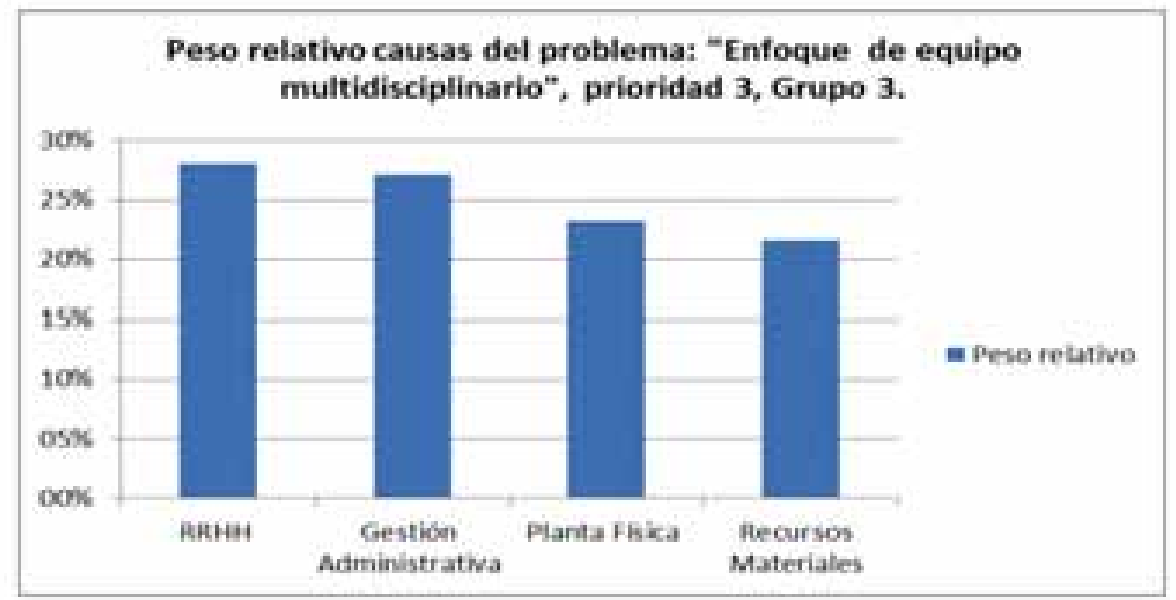

Fuente: Elaboración propia. Diplomado Enfoques y Nuevas Perspectivas para la Atención Primaria de Salud, (20I0).

GRUPO D. Las áreas más críticas de la APS para este grupo fueron: $1^{\circ}$ Incompatibilidad de dos Modelos en APS, $2^{\circ}$ Mala gestión del SOME y $3^{\circ}$ Trabajo en equipo.

$1^{\circ}$ Incompatibilidad de dos Modelos en APS (Gráfico $\mathrm{N}^{\circ} \mathrm{I} 0$ ), se explica por las siguientes causas: Gestión de política Pública (33\%), RRHH (30\%), Infraestructura (I8\%), Modelo de Salud Pública (10\%), Modelo de Salud Familiar (10\%).

Gráfico $N^{\circ} 10$ :

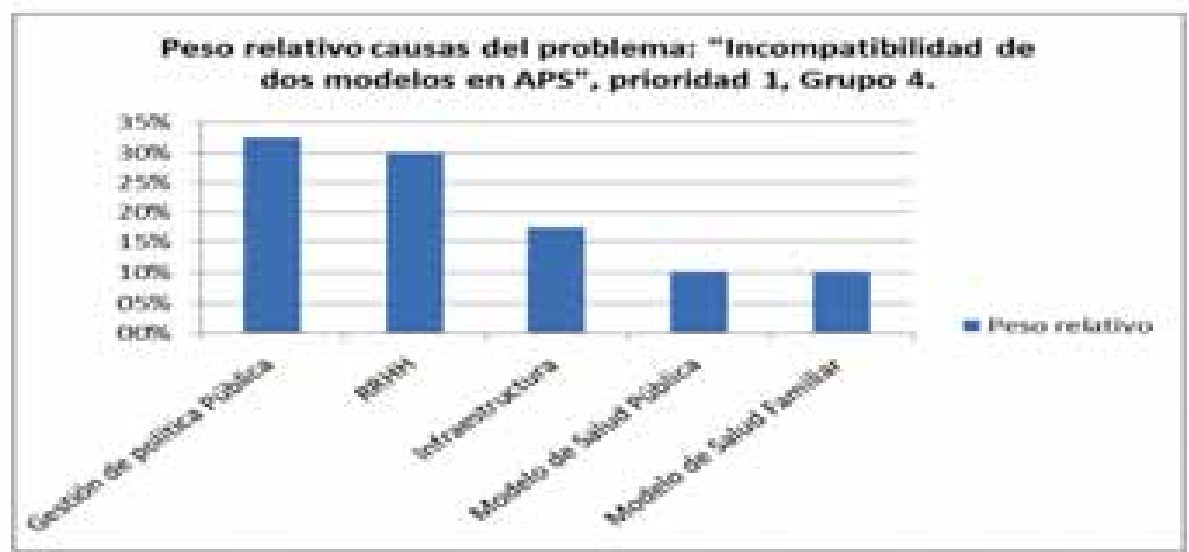

Fuente: Elaboración propia. Diplomado Enfoques y Nuevas Perspectivas para la Atención Primaria de Salud, $(2010)$. 
$2^{\circ}$ Mala gestión del SOME (Gráfico $N^{\circ} \mathrm{II}$ ), se explica por las siguientes causas: Gestión del Dpto. de Salud Municipal (25\%), RRHH (24\%), Gestión Directiva (18\%), Capacitación (I8\%), Infraestructura (15\%).

\section{Gráfico $\mathrm{N}^{\circ}$ II:}

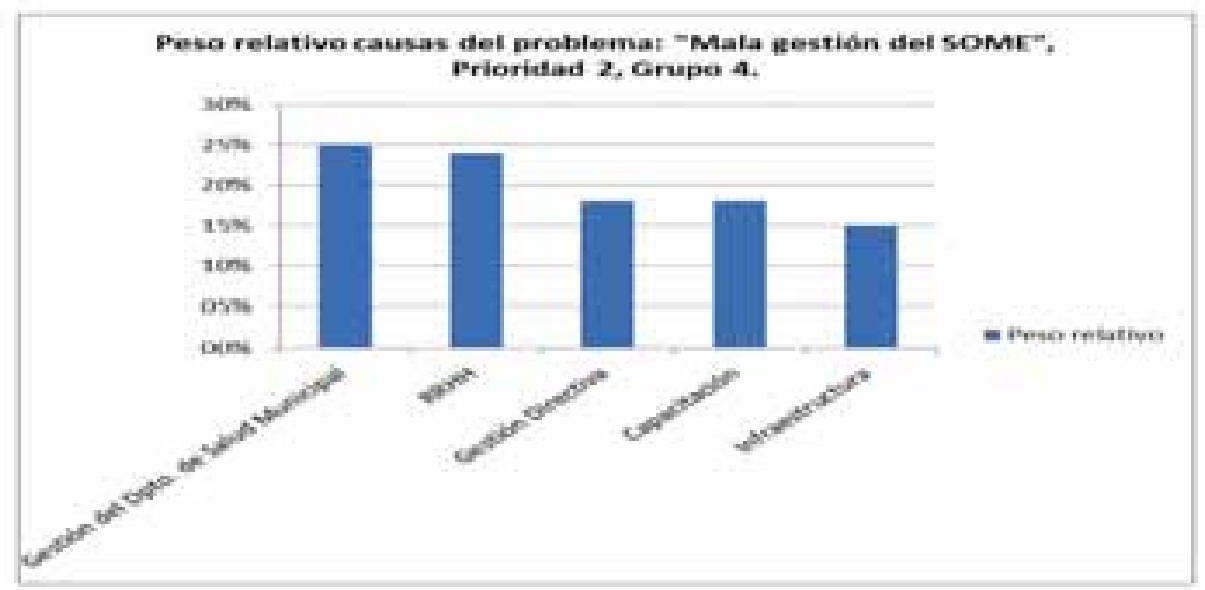

Fuente: Elaboración propia. Diplomado Enfoques y Nuevas Perspectivas para la Atención Primaria de Salud, (20I0).

$3^{\circ}$ Trabajo en equipo (Gráfico $\mathrm{N}^{\circ} \mathrm{I}$ ), se explica por las siguientes causas: $\mathrm{RRHH}(25 \%)$, Gestión Directiva (24\%), Cuidados del Equipo (23\%), Infraestructura y Recursos Tecnológicos (14\%), Capacitación (14\%).

\section{Gráfico N²:}

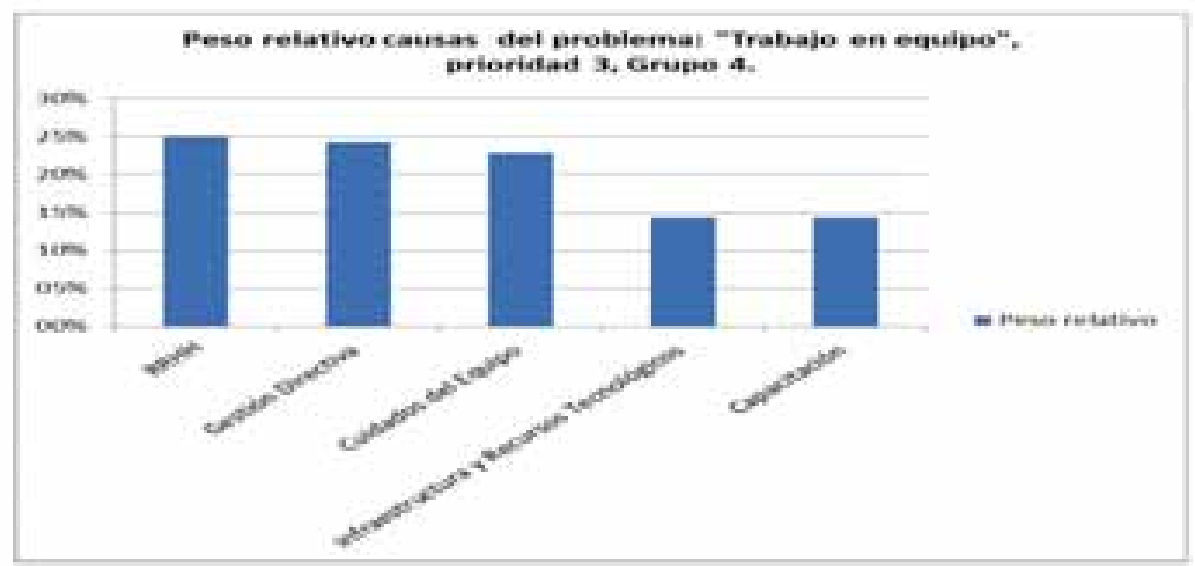

Fuente: Elaboración propia. Diplomado Enfoques y Nuevas Perspectivas para la Atención Primaria de Salud, (20I0). 
GRUPO E. Las áreas más críticas de la APS para este último grupo fueron: $I^{\circ}$ Descripción de Procesos, $2^{\circ}$ Clima Organizacional y $3^{\circ}$ Cultura Organizacional.

$1^{\circ}$ Descripción de Procesos (Gráfico $\mathrm{N}^{\circ} \mathrm{I3}$ ), se explica por las siguientes causas:Técnico Administrativo (4l\%), RRHH (3I\%), Factores externos a la institución (I8\%), Método (10\%).

Gráfico $N^{\circ}$ 13:

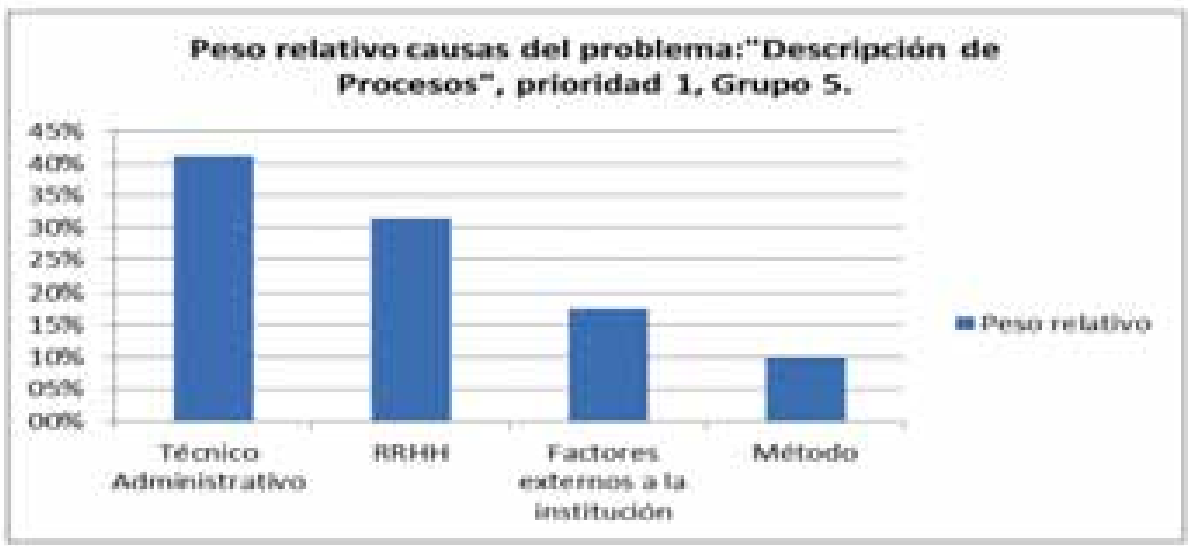

Fuente: Elaboración propia. Diplomado Enfoques y Nuevas Perspectivas para la Atención Primaria de Salud, (2010).

$2^{\circ}$ Clima Organizacional (Gráfico $\mathrm{N}^{\circ} \mathrm{I4}$ ), se explica por las siguientes causas: Personas (46\%), Gestión (37\%), Infraestructura (10\%), Equipo (6\%).

\section{Gráfico $\mathrm{N}^{\circ}$ 14:}

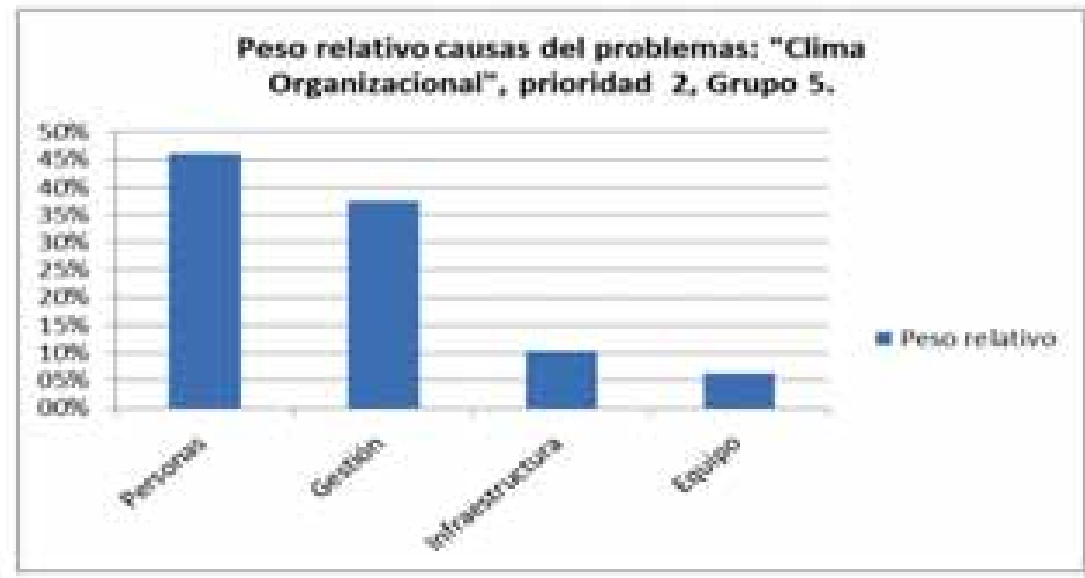

Fuente: Elaboración propia. Diplomado Enfoques y Nuevas Perspectivas para la Atención Primaria de Salud, (20I0). 
$3^{\circ}$ Cultura Organizacional (Gráfico $N^{\circ} 15$ ), se explica por las siguientes causas: Personas (47\%), Gestión (29\%), Equipo (16\%), Institución (8\%).

\section{Gráfico N¹5:}

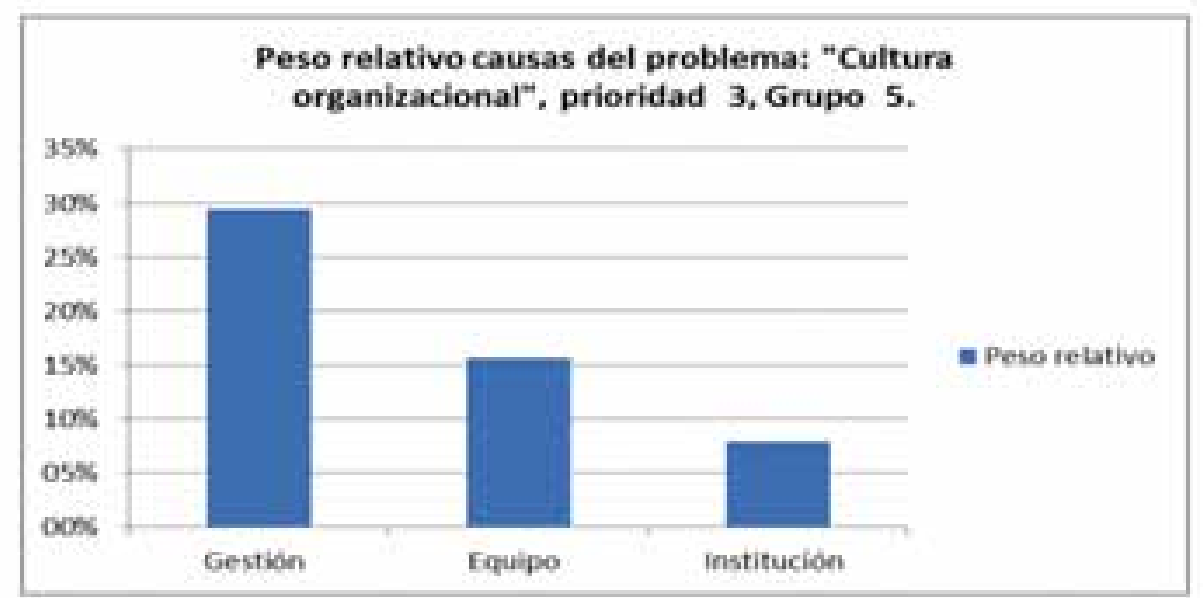

Fuente: Elaboración propia. Diplomado Enfoques y Nuevas Perspectivas para la Atención Primaria de Salud, (2010).

Diagrama $\mathrm{N}^{\circ}$ I: Una visión global del resultado de los trabajos grupales, reflejado en los Diagramas de Causa y Efecto (Ishikawa) 2223

22.- En este Mapa conceptual está la síntesis del trabajo realizado por los grupos. En cada rama, correspondiente a cada grupo de trabajo, hay tres áreas problema, dispuestas en orden de priorización. Dentro de cada área problema están las causas del mismo, con sus respectivos pesos relativos dentro del área problema.

23.- Para la realización del Mapa Conceptual se usó como insumo el resultado de las Matrices de riesgo de cada grupo de trabajo y para su representación gráfica el software FreeMind. 


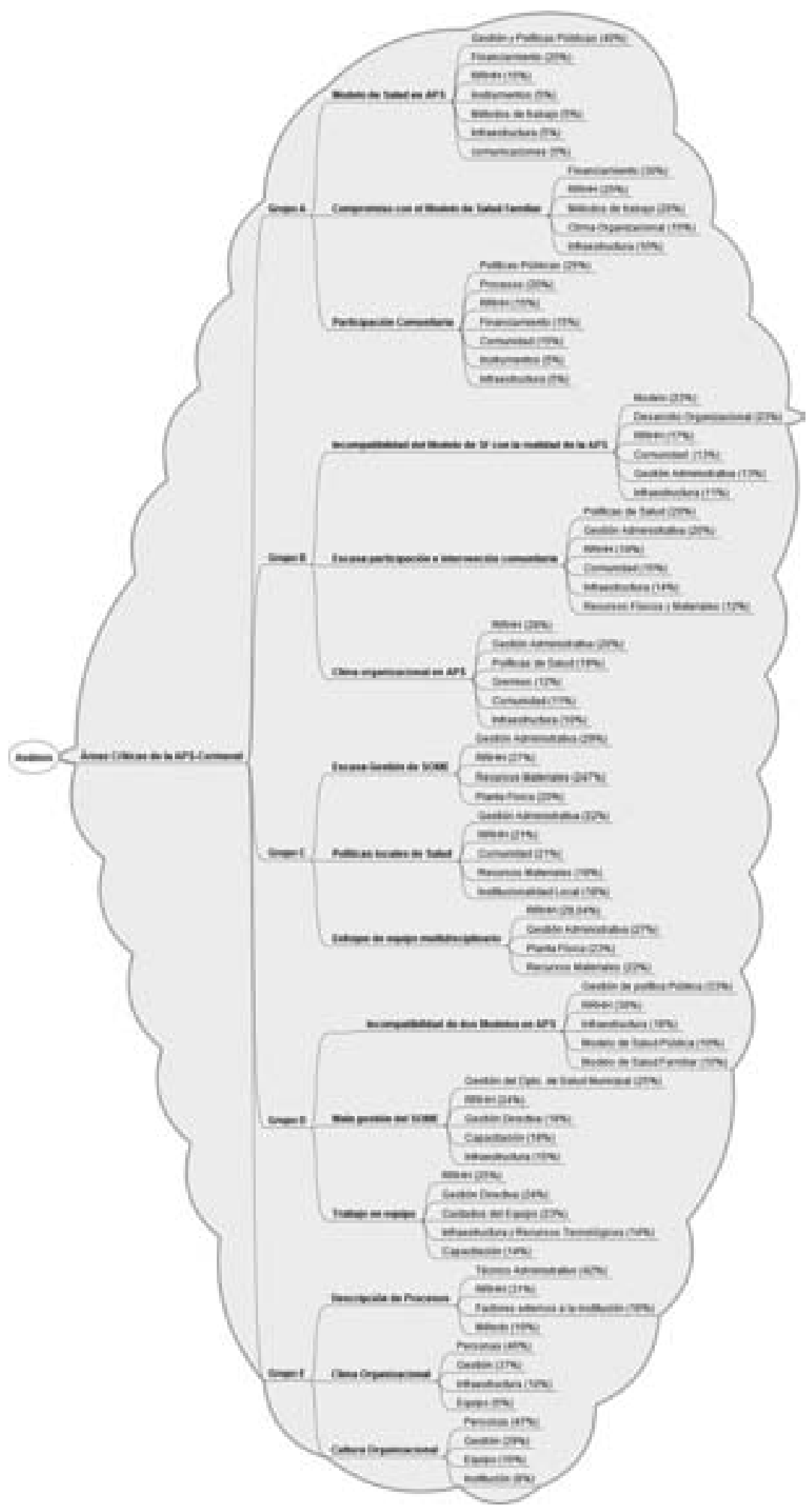


Tabla $N^{\circ} 2$

Resultados generales de los Diagramas de Causa y Efecto (Ishikawa) ${ }^{24}$

\begin{tabular}{|c|c|c|c|c|}
\hline 0008 & Irings & 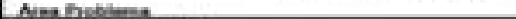 & 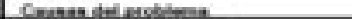 & Fetsintat: \\
\hline 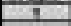 & 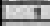 & 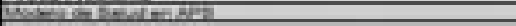 & mons & $-\infty$ \\
\hline \pm & 1 & 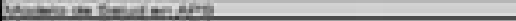 & 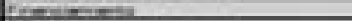 & $\tan x$ \\
\hline $\mathrm{E}$ & 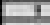 & 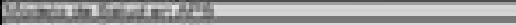 & $5=5$ & ats \\
\hline & & 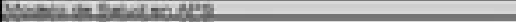 & 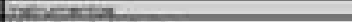 & 37 \\
\hline 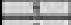 & & 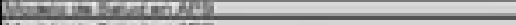 & 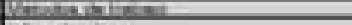 & 30 \\
\hline 9 & -1 & 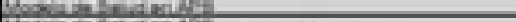 & Etrantoation & 5 \\
\hline 1 & 1 & 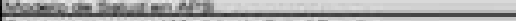 & 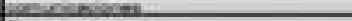 & 50 \\
\hline 2 & a. & 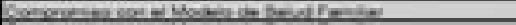 & 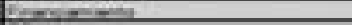 & 20 \\
\hline ב & & 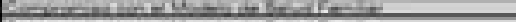 & 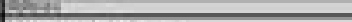 & 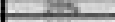 \\
\hline \pm & $t$. & 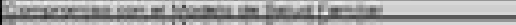 & 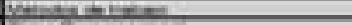 & 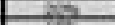 \\
\hline \pm & 2 & 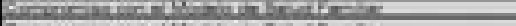 & 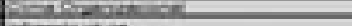 & Tis \\
\hline \pm & 2 & 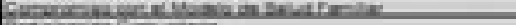 & Fankicica & 23 \\
\hline 1 & I & 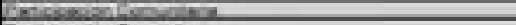 & 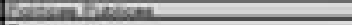 & 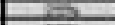 \\
\hline \pm & 3 & 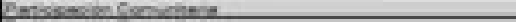 & Comatral & 20 \\
\hline 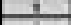 & 1 & E-3bont & Eveng & 58 \\
\hline$\div$ & +1 & 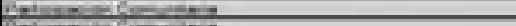 & 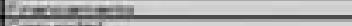 & $\rightarrow 2$ \\
\hline I & 1: & 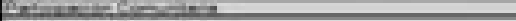 & Larmin & $\cos 5$ \\
\hline 1 & 3 & 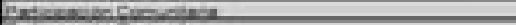 & 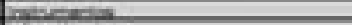 & +2 \\
\hline$t^{2}$ & 21 & 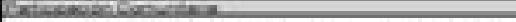 & 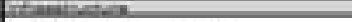 & 28 \\
\hline 2 & + & 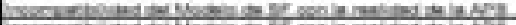 & Sooking & mo \\
\hline I & I & 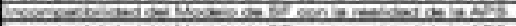 & 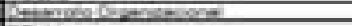 & 20 \\
\hline 1 & 1 & 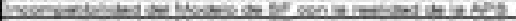 & Daten & $\tan$ \\
\hline 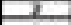 & 3 & 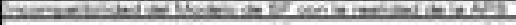 & $x_{m=1}=1$ & men \\
\hline 2. & 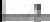 & 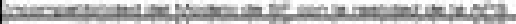 & 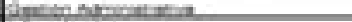 & 572 \\
\hline 2 & 1 & 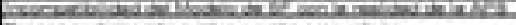 & Fancasa & 205 \\
\hline 2 & 3 & 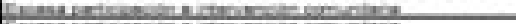 & 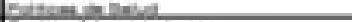 & 3 \\
\hline 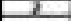 & 2 & 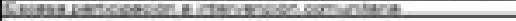 & 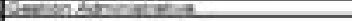 & 200 \\
\hline 2 & 3 & 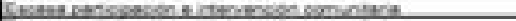 & Dentin & the ras r \\
\hline 2 & t & 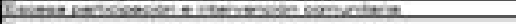 & 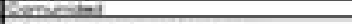 & Es \\
\hline 1 & $\mathbf{z}$ & 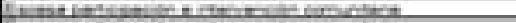 & 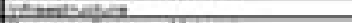 & 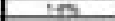 \\
\hline 2 & & 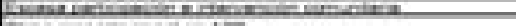 & - - & 90 \\
\hline 2 & 2 & 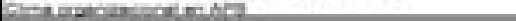 & fonets & S. \\
\hline x & e & 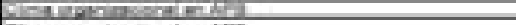 & 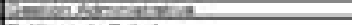 & $\mathrm{ns}$ \\
\hline 2 & 1 & 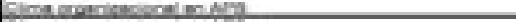 & 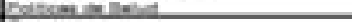 & $1 \%$ \\
\hline e & 1 & 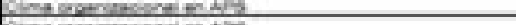 & Dernioge & 30 \\
\hline 2 & 1 & 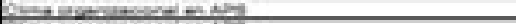 & Cansolet & 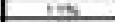 \\
\hline 1 & 1 & 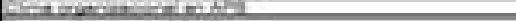 & Hantentex & $\cos =$ \\
\hline ? & 1 & 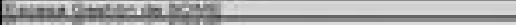 & 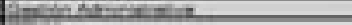 & 20 \\
\hline 2 & 1 & 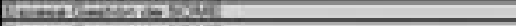 & Lestrong a & 205 \\
\hline 3 & 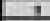 & 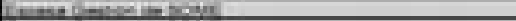 & 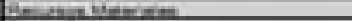 & $\tan$ \\
\hline 2 & T & 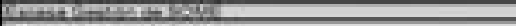 & Senteise & 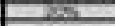 \\
\hline 3 & 2 & 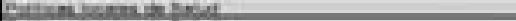 & 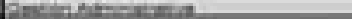 & 200. \\
\hline 21 & $2 \mathrm{z}$ & 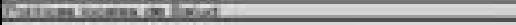 & F $=\mathrm{F}^{\mathrm{n}}$ & etent \\
\hline 7 & 2 & 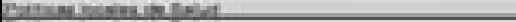 & Campitest & $x=$ \\
\hline 3 & 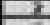 & 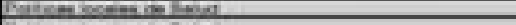 & 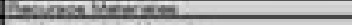 & 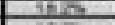 \\
\hline 2 & 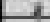 & 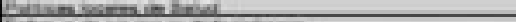 & 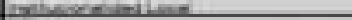 & 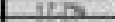 \\
\hline 3 & 1 & 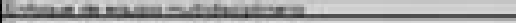 & Fostis & $=10$ \\
\hline 3 & 3. & 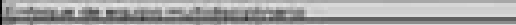 & 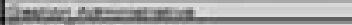 & 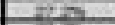 \\
\hline 2E & I & 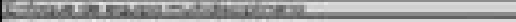 & 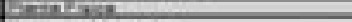 & $3-2-5$ \\
\hline 3 & 1 & 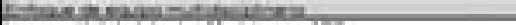 & 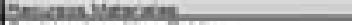 & 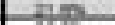 \\
\hline a & $\frac{1}{2}$ & 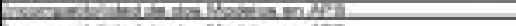 & 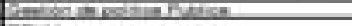 & $2 \times 15$ \\
\hline 4 & 1 & 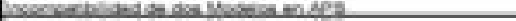 & 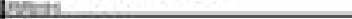 & 2000 \\
\hline 4 & 1 & 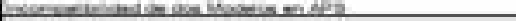 & athantionsol & $12=$ \\
\hline 4 & 1 & 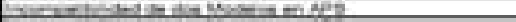 & 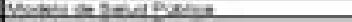 & $=50$ \\
\hline+1 & 9 & 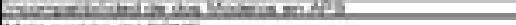 & 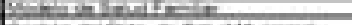 & Was \\
\hline-1 & 4 & 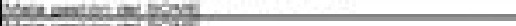 & 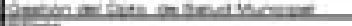 & 20 \\
\hline i & 2. & 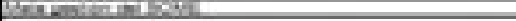 & 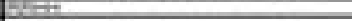 & 200.5 \\
\hline 4 & a. & 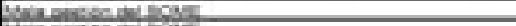 & 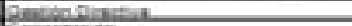 & 390 \\
\hline 4 & ㄴ. & 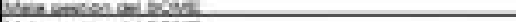 & phatowes & then \\
\hline 4 & 2 & 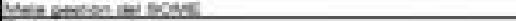 & Ghantsons & 1509 \\
\hline 4 & T) & 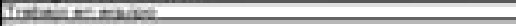 & Eater & 805 \\
\hline 4 & 2 & forltedestages & 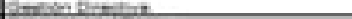 & 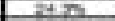 \\
\hline ta & I & 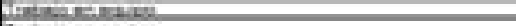 & 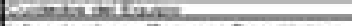 & .Mt \\
\hline A & 1 & 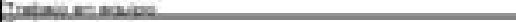 & 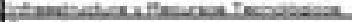 & $31=$ \\
\hline 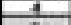 & 1 & 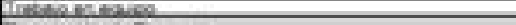 & 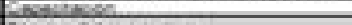 & $\operatorname{trns}$ \\
\hline 2 & 1 & 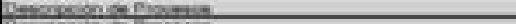 & 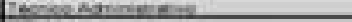 & 410 \\
\hline 3 & 3 & 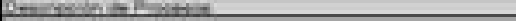 & 50 & (2n-4) \\
\hline 3 & 1 & 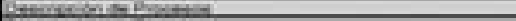 & 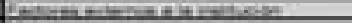 & itha \\
\hline $3=$ & 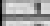 & 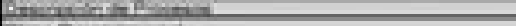 & Desten & $201 / 2$ \\
\hline 2 & 2 & 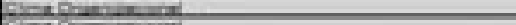 & Cenacta. & +6 \\
\hline 2 & 2 & 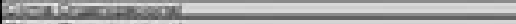 & $2 \min 20$ & aren \\
\hline 2 & 2 & 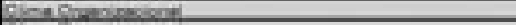 & 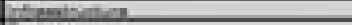 & 30 \\
\hline 3 & 2 & 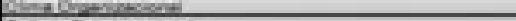 & 10000 & 20 \\
\hline 3 & 1 & 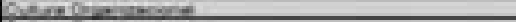 & $D=m$ & $\operatorname{men}$ \\
\hline 3 & 1 & 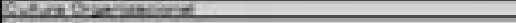 & 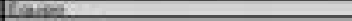 & $\cos$ \\
\hline 2 & 3. & 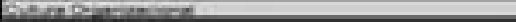 & 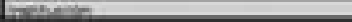 & 200 \\
\hline
\end{tabular}

Fuente: Elaboración propia. Diplomado Enfoques y Nuevas Perspectivas para la Atención Primaria de Salud, (2010).

24.- Ordenados dentro de cada grupo por área problema. 
Gráfico $N^{\circ}$ 16. Visión agregada de las áreas críticas priorizadas y causas de las mismas. ${ }^{25}$

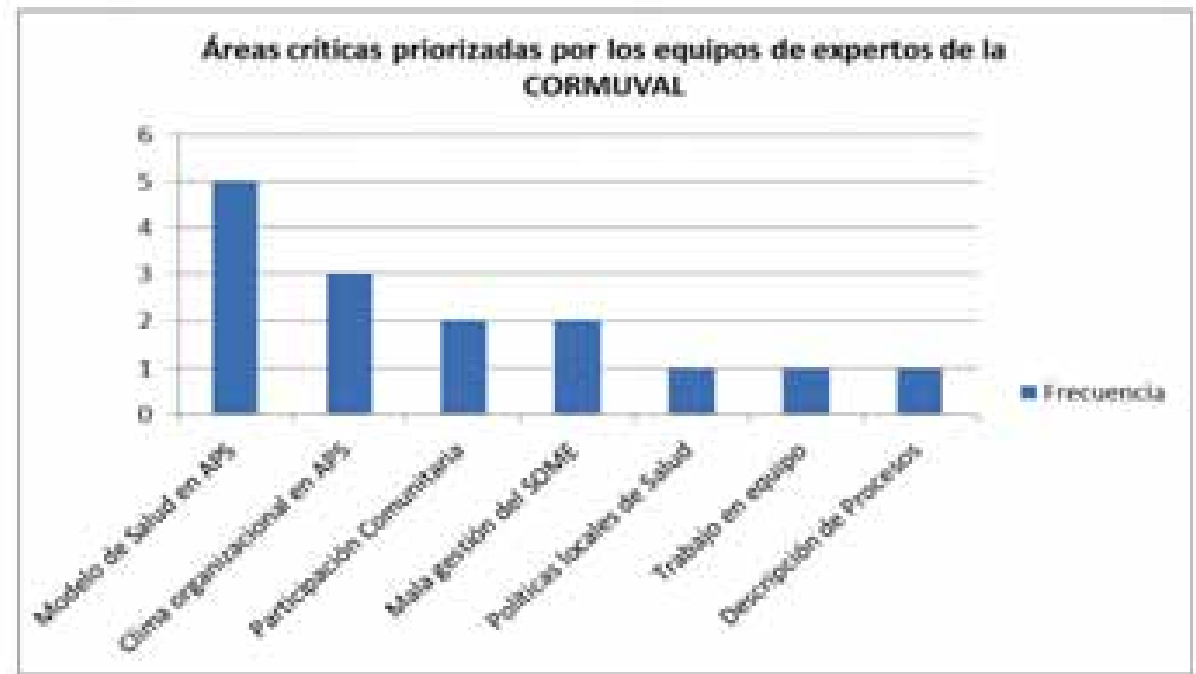

ruente: Elaboracion propia. Diplomado tntoques y Nuevas rerspectivas para la Atencion Primaria de Salud, (2010).

Gráfico $N^{\circ}$ I7. Principales causas de las áreas problema en APS, según Panel de expertos de la Cormuval

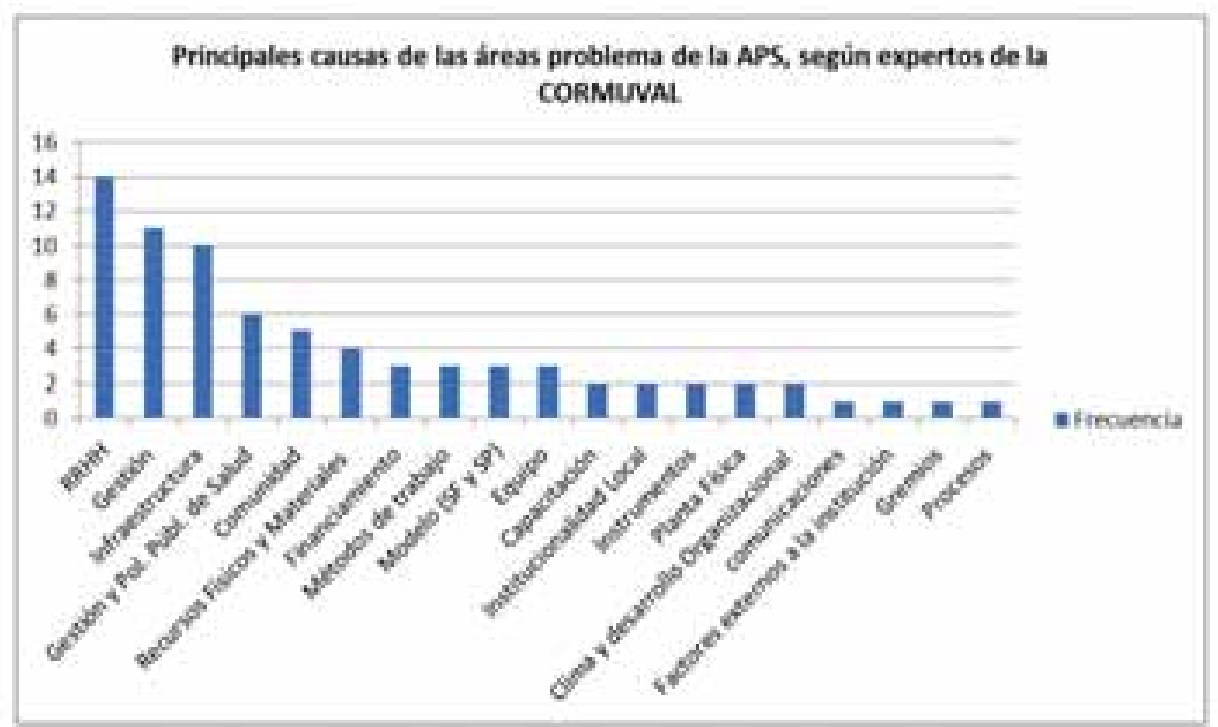

Fuente: Elaboración propia. Diplomado Enfoques y Nuevas Perspectivas para la Atención Primaria de Salud, (20I0).

25.- Representa la frecuencia con que aparecen las áreas problema, priorizadas, y las causas de los distintos problemas priorizados. 


\section{Discusión}

La Reforma de Salud en Chile, vigente desde la promulgación de la Ley de Autoridad Sanitaria y de Gestión en Red el año 2004, explícitamente señala que los cambios esperados para lograr los objetivos sanitarios propuestos dependen, entre otros, del trabajo en red centrado en la Atención Primaria de Salud (APS) y basado en un modelo de Atención Integral de Salud con Enfoque Familiar.

Los resultados obtenidos en los Centros de Salud y Consultorios estudiados, después de poco de más de seis años de iniciada la Reforma, señalan que aún persisten problemas importantes en la implementación y apropiación del modelo por parte de los equipos de salud, una escasa participación de la comunidad y en problemas organizativos internos de los establecimientos de salud.

Respecto a la identificación de problemas, es significativo que algunos grupos de participantes señalen que los problemas más críticos y relevantes del Centro de Salud tienen que ver precisamente con el modelo de salud definido o con el compromiso que los equipos de salud tienen con el mismo. Incluso algunos de ellos señalan la incompatibilidad de modelo de salud familiar con la realidad de la APS. Si bien se señalan diferentes causas que explicarían este fenómeno, tales como debilidades en la gestión de las políticas públicas, financiamiento, infraestructura o desarrollo organizacional, el hecho significativo es que a juicio de estos actores se estaría poniendo en tela de juicio aquello que está en la base y sustenta el proceso de reforma.

Habría en este sentido una falta de diálogo entre quienes diseñan y planifican la política pública (entidades rectoras y normativas del nivel central) y quienes son los encargados de implementarla (instituciones y profesionales prestadores de salud). Por otra parte, se percibe una cierta contradicción o incompatibilidad entre el cumplimiento de metas o resultados de producción de servicios, frecuentemente ligados a la entrega de recursos financieros e incentivos al cumplimiento, y la realización del modelo de atención. Es por ello que la explicación de las causas de las dificultades para la implantación y el compromiso con el modelo de salud familiar, fueron relacionados especialmente con la generación de las políticas públicas y con el sistema de financiamiento, y no, como se podría haber esperado, con problemas relacionados con la gestión clínica (métodos de trabajo) o con recursos (tales como falta de profesionales o infraestructura).

Otro hallazgo que debe llevar a la reflexión dice relación con la participación comunitaria. Efectivamente, algunos de los equipos de salud perciben como un factor crítico del Centro de Salud la escasa participación e intervención de la comunidad, la cual se mantiene en un rol predominantemente demandante de servicios, pero que no se hace parte de la solución de los mismos. Aquí, nuevamente, el peso mayor de las causas señaladas dicen relación con la gestión de las políticas públicas y la gestión administrativa, lo que lleva a pensar que esta situación no va a cambiar a menos que exista una intención clara por parte de las autoridades sectoriales por modificarla.

También se requiere mayor investigación y adecuación de los modelos de participación comunitaria, en concordancia con los cambios ocurridos en la sociedad chilena. Debieran 
revisarse los canales, mecanismos y significados de participación social que se utilizan; muchas veces éstos responden más a los paradigmas y modelos históricamente utilizados por los equipos de salud que a las expectativas, formas de expresión y significados de su relación con el Estado que hoy tienen los ciudadanos. De allí que el mayor peso de la escasa participación se atribuya a causas relacionadas con las políticas de salud y la gestión administrativa y técnica, antes que con aquellas relacionadas con la disponibilidad de recursos.

Respecto a los problemas identificados con la organización y administración interna de los Centros de Salud, ellos se dividen en problemas relacionados con la gestión del personal (clima organizacional, trabajo en equipo, cultura organizacional) y en la gestión de los Servicios de Orientación Médica y Estadística (SOME). Este último aspecto es igualmente relevante en la gestión de la red interna y externa del Centro de Salud, la cual no sólo puede ser una causal importante de insatisfacción por parte de la población con la calidad global de los servicios recibidos, sino que también puede perturbar de forma importante la adecuada referencia y contrareferencia dentro de la red asistencial.

Siendo el componente asistencial de la atención de salud fuertemente dependiente de la relación entre prestador y usuario, la sensación de desgaste de los recursos humanos que se advierte en el estudio, requiere una especial preocupación por el bienestar y salud integral de los propios trabajadores; sería difícil esperar una mejoría en la satisfacción del usuario si no se implementan políticas y programas orientadas a preparar al personal de salud para trabajar en situaciones de alta presión y demanda (autocuidado).

Probablemente también hay que profundizar en los aspectos relacionados con la gestión de la demanda por atención médica, el manejo de las agendas médicas para la atención diferida o el enfrentamiento de los tiempos de espera y eliminación de las llamadas colas o filas para conseguir hora de atención médica.

Tan relevante como los problemas que se identifican por parte de los participantes del estudio, pueden ser aquellos temas o problemas que no fueron identificados o visualizados como tales, y que sin embargo constituyen componentes fundamentales de la agenda de las políticas públicas.Así, por ejemplo, llama la atención la ausencia de mención de ámbitos tales como la calidad de la atención de salud y componentes derivados de la misma, como la satisfacción del usuario con el trato recibido o con la relación proveedor usuario.

Finalmente, cabe mencionar que el tipo de problemas menos significativos señalados por el personal de salud son aquéllos relacionados con la infraestructura y los recursos físicos y materiales. Esto contrastaría con el énfasis y acento de la inversión pública en infraestructura, en desmedro de aquella que se realiza en los recursos humanos. Sin desmerecer la importancia que tiene dotar de recursos físicos y tecnológicos adecuados y modernos a los servicios de salud, habría que poner igual énfasis y preocupación por los aspectos relacionales en la atención sanitaria y legitimación de las políticas públicas ante los funcionarios de salud y la población en general. 


\section{Conclusión}

Las políticas de salud para la Atención Primaria de Salud en Chile se centran principalmente en el logro de metas específicas, que para muchos municipios se deben realizar con recursos materiales y humanos escasos. Esta diada ha creado una gran presión de trabajo en los equipos de salud, impidiendo la creación de espacios de reflexión donde los equipos sean capaces de observar cómo realizan su trabajo y si es necesario hacer cambios o desarrollar nuevas estrategias. Entendemos que es responsabilidad de los equipos dar a conocer sus necesidades y falencias ante una comunidad altamente demandante por mayor y mejor atención.

El desarrollo de un programa de formación acción donde los equipos de salud encuentran y crean por sí mismos sus espacios de reflexión, fortalece el desarrollo de un análisis crítico respecto a su entorno, interno y externo. Respecto al análisis externo, los estudios e investigaciones en APS se centran principalmente en el usuario externo y sus necesidades, no abordando la relación directa entre los equipos de salud, el cumplimiento de metas y los grados de satisfacción que estos equipos alcanzan en el camino hacia el cumplimiento de dichas metas.

Por otra parte, la detección de problemas estructurales del sistema de atención primaria de salud en Chile y las reales causas de estos problemas, bajo la percepción cualitativa de los equipos locales, permite la determinación de una línea base para el desarrollo de proyectos de intervención específicos en cada una de las áreas del desarrollo local y comunitario. Prueba de ello es que dentro del esquema de priorización de problemas, analizados por el grupo de participantes de esta experiencia, se destaca que entre los motivos prioritarios de satisfacción personal se encuentra el buen ambiente laboral y particularmente el desarrollo de recursos humanos.

Como contrapunto a lo señalado, en la medida que los equipos locales desarrollen intervenciones específicas, independiente del área a intervenir, será la propia comunidad la que se beneficiará con la intervención realizada.

El desarrollo del programa de formación acción, llevado a cabo en esta oportunidad en la Corporación Municipal de Valparaíso, viene a materializar el esfuerzo que el ámbito académico debe desarrollar para contribuir al fortalecimiento de la APS en Chile. La creación de espacios de reflexión y de análisis como éste contribuye al desarrollo de una masa crítica de trabajadores y trabajadoras que ve y siente que es aquí, en este espacio de la APS, donde se deben centrar los esfuerzos organizacionales, una demanda para que los policy makers aborden los nuevos desafíos que la Atención Primaria de Salud en Chile necesita desarrollar.

\section{Agradecimientos}

El presente artículo es parte integral del desarrollo académico de los autores. Se agradece en forma particular a la Escuela de Salud Pública de la Universidad de Chile por la oportunidad de realizar esta experiencia innovadora en la ciudad de Valparaíso, potenciando su rol de acercamiento a la realidad sanitaria nacional y particularmente a los equipos de trabajo de la atención primaria de salud. 
Bibliografía

Armas, R., Torres, A., Arriagada, J., Muñoz, F., Salinas, R., \& Crocco, P. (20I0). Proceso para priorizar las líneas de investigación esencial de interés para el Ministerio de Salud de Chile. Revista Médica de Chile, 40 I-405.

Canales, M., (Coordinador-editor). (2006). Metodologías de Investigación Social. Santiago de Chile: LOM Ediciones.

Capponi, R. (2004). El amor después del amor (2a. ed.). Santiago de Chile: Ed. Grijalbo. Ellis, R. (2004). Modelo de Ajuste de Riesgo. Modelo de Atención en Salud. Santiago de Chile: Ministerio de Salud.

Hernández, R., Fernández-Collado, C., \& Baptista, P. (2008). Metodología de la investigación. En R. Hernández, C. Fernández-Collado, \& P. Baptista, Metodología de la investigación (págs. 34I-35I). México D.F.: McGraw-Hill/Interamericana Editores, S.A.

Hidalgo, C. C. (1999). Salud Familiar: un modelo de atención integral en la atención primaria. Santiago de Chile: Ediciones Universidad Católica de Chile.

Hopkins, J. (1998). Cost of Health. New York: The John Hopkins School of Hygiene and Public Health.

Konow, I., \& Pérez, G. (1990). Método Delphi. En H. Acuña, I. Konow, \& (compiladores), Métodos y técnicas de investigación prospectiva para la toma de decisiones (págs. 7-32). Santiago: Fundación de Estudios Prospectivos, Planificación Estratégica y Decisiones de Alto Nivel de la Universidad de Chile.

Martínez O., L. (1999). Justicia y Medicina Pública. Del principio ético a los desafíos en su aplicación. Santiago de Chile: LOM Ediciones.

Martín-Zurro, A. (1993). Manual de Atención Primaria (2a. Edición ed.). Madrid: Doyma Ediciones.

Ministerio de Salud. (198I). Anales Ministerio de Salud. Servicio Nacional de Salud. Santiago de Chile: Ministerio de Salud.

Ministerio de Salud. (2002). Objetivos Sanitarios y Modelo de Salud para la Década 200020I0. Santiago de Chile: Documento Oficial. Ministerio de Salud.

Ministerio de Salud, Depto. APS. (2002). Salud Familiar: Período 1990-2002. Santiago de Chile: Departamento Atención Primaria. Ministerio de Salud.

OMS. (1978). Conferencia Internacional de Alma-Ata. Ginebra: Organización Mundial de la Salud.

Sautu, R., Boniolo, P., Dalle, P., \& Elbert, R. (20I0). Manual de Metodología. Buenos Aires: Prometeo Libros. 
Scribano, A. O. (2008). El proceso de Investigación social cualitativo. Buenos Aires: Prometeo Libros.

Secretaría Técnica Asesora para el FSS, Ministerio de Salud. (2009). Insumos para un plan de fortalecimiento del sector salud. Santiago: Ministerio de Salud. 